\title{
Interval data aggregation technology for large scale decision making
}

\author{
Linxin Chen ${ }^{\mathrm{a}, \mathrm{b}}$, Riqing Chen ${ }^{\mathrm{b} *}$, Jian $\operatorname{Lin}^{\mathrm{a} *}$ \\ ${ }^{a}$ College of Computer and Information Sciences, Fujian Agriculture and Forestry University, Fuzhou 350002, \\ China \\ b The Digital Fujian Research Institute of Big Data for Agriculture and Forestry, Fujian Agriculture and Forestry \\ University, Fuzhou 350002, China
}

\begin{abstract}
In this paper, an improved multiple attribute decision making (MADM) method based on the proposed novel score function and accuracy function of interval-valued intuitionistic fuzzy numbers (IVIFNs) is proposed to aggregate large-scale data. The attribute values in the decision matrices provided by each decision-maker (DM), which are characterized by interval numbers. First, a transformation matrix is introduced to define the concepts of satisfactory set, unsatisfactory set and uncertainty set of alternatives. An approach is then developed for aggregating attribute values into IVIFNs, and we will obtain the collective evaluation of each alternative. Next, using the interval-valued intuitionistic fuzzy weighted averaging operator, the collective attribute values characterized by IVIFNs are aggregated to get the overall evaluation of alternatives. The score function and accuracy function are applied to calculate the score degree and the rank of each alternative. Finally, a large-scale example is given to verify the validity of the reported method.
\end{abstract}

Keywords: decision making; interval-valued intuitionistic fuzzy number; aggregation technology; accuracy function; score function.

\section{Introduction}

The explosive increase of information has become an important turning point for decision analysis. Lots of previously inaccessible data is readily available in today's digital era, and some seemingly unrelated data can be of great value when combined. People desire to obtain some regular knowledge from the uncertainty to support decision-making. However, there are many uncertainties in the actual large-scale data environment, and the collected data are often noisy, inaccurate, or even incomplete.

Fuzzy set [1] is a powerful mathematical tool for dealing with uncertainty in the decisionmaking environment. Its effectiveness has been proven in various application fields, and it is one of the research hotspots in intelligent computing theory and its applications [2-4]. Atanassov [5,6] introduced the intuitionistic fuzzy set(IFS) [5,7], which is a generalization of fuzzy set [1] and more suitable for dealing with fuzziness and uncertainty than the ordinary fuzzy set (In fact, it

* Corresponding author. E-mail addresses: chenlinxin97@gmail.com (L.X. Chen), riqing.chen@fafu.edu.cn (R.Q. Chen), linjian36@163.com (J. Lin). 
may still be difficult to determine the crisp values of the membership and non-membership of an element to the set. In this case, a range of values may be a more appropriate metric to adapt to ambiguity.). Then, Atanassov [8] further extends the intuitionistic fuzzy sets and generalized the concept of interval intuitionistic fuzzy sets. IVIFSs have proven to be very useful in dealing with uncertainty and ambiguity. Researchers have done a lot of work to develop and enrich the theory of IVIFS, which has paved the way for our analysis and decision-making in a large-scale data environment.

One of the active research topics is investigating multi-attribute decision-making by how to rank various types of data, especially interval-valued intuitionistic fuzzy numbers(IVIFNs), which contains three parts of information, membership degree, non-membership degree, and hesitation degree. The IVIFN describes the actual evaluation of decision-makers more accurately, but there is no unified ranking method yet. Xu [9] first proposed the concept of score function and accuracy function of interval intuitionistic fuzzy numbers, which are used to rank interval-valued intuitionistic fuzzy numbers(IVIFNs). In recent years, many scholars have explored the information of interval intuitionistic fuzzy numbers and proposed different score functions or accuracy functions [10-15], Gao [16] proposed the score function from the perspective of hesitancy, Wang [17] proposed a new score function of IVIFVs to develop an improved MADM method, Gong [18] analyzed the shortcomings of some existing score functions and accuracy functions and constructed new score function and accuracy function using the idea of the full probability formula of probability theory. To overcome the drawbacks of the score functions of IVIFNs presented by Xu [9] and Kumar [19], Chen [20] propose a new MADM method based on the Beta distribution, expected values of intervals and a new score function of IVIFNs. However, after comparing and analyzing the score functions and accuracy functions constructed in this literature, we find that they still have the phenomenon of sorting failure.

Another active research topic is investigating multi-attribute decision-making by aggregating data information characterized by crisp numbers, intervals, or other types of values provided by individuals into the overall evaluation information of the alternatives. However, few researchers have focused on transforming the attribute information of alternative into interval-valued intuitionistic fuzzy numbers to complete decision-making. Yue [21] defined the concepts of supporting, opposing, and neutral sets of alternative, respectively, and proposed a method for transforming attribute values characterized by crisp numbers into IFN. Introduced the concepts of satisfaction interval and dissatisfaction interval, Yue [22] developed an approach for aggregating attribute into the collective attribute interval-valued intuitionistic fuzzy number (IVIFN). Yue [23] presented soft computing to aggregate all individual values on the decision matrix into an IVIFN. Lin [24] pointed out that Yue's aggregation method is unreasonable in some cases. Considering the internal connection of the decision-makers' educational background, political status, and personal preferences, Lin established an improved method to aggregate crisp values into intuitionistic fuzzy numbers for group decision making. Wan [25] and Xu [26] proposed a 
new and practical method for aggregating real numbers to AIFN in a heterogeneous MAGDM environment, respectively.

This paper focuses on a novel aggregation method to deal with large-scale data MADM problems to overcome the aforementioned main difficulties and limitations. The main innovations and contributions are as follows: (1) define novel score function and accuracy function for better ranking of IVIFNs; (2) an aggregation technology, based on the transformation method, to aggregate interval data into interval-valued intuitionistic fuzzy information for MADM is proposed; (3) a new MADM method is developed to solve the large-scale data MADM problems in which the decision information is expressed as interval numbers.

The remainder of this paper is organized as follows. Section 2 reviews some basic concepts of IVIFNs, IVIFSs, and some existing score and accuracy functions of IVIFNs. In Section 3, we briefly review Yue's MADM method [27] and use two counter-examples to illustrate his method's drawbacks. Section 4 presents a new MADM method based on the proposed score and accuracy functions. In Section 5, a practical example is given to illustrate the rationality of the proposed method. Section 6 compares the proposed score and accuracy functions with others. Furthermore, this paper ends in Section 7 with the Conclusions.

\section{Preliminaries}

In this section, we introduce some of the basic concepts of the interval-valued intuitionistic fuzzy set(IVIFS) and interval-valued intuitionistic fuzzy number(IVIFN).

Definition 2.1. [8] Let $X=\left\{x_{1}, x_{2}, \ldots, x_{n}\right\}$ be a discourse. An interval-valued intuitionistic fuzzy set $\tilde{A}$ in $X$ is represented by $\tilde{A}=\left\{<x_{i}, \mu_{\tilde{A}}\left(x_{i}\right), v_{\tilde{A}}\left(x_{i}\right)>\mid x_{i} \in X\right\}, \quad \mu_{\tilde{A}}(x)$ and $v_{\tilde{A}}(x)$ denote the membership degree and the non-membership degree of element $x \in X$ belonging to the IVIFS $\tilde{A}$, respectively. $\mu_{\tilde{A}}\left(x_{i}\right)=\left[\mu_{\tilde{A}}^{-}\left(x_{i}\right), \mu_{\tilde{A}}^{+}\left(x_{i}\right)\right]$,

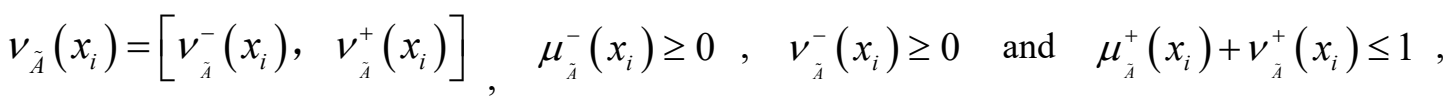
$i=1,2, \ldots, n$. The hesitation degree of $x$ to the IVIFS $\tilde{A}$ is denoted by $\pi_{\tilde{A}}\left(x_{i}\right)=\left[1-\mu_{\tilde{A}}^{+}\left(x_{i}\right)-v_{\tilde{A}}^{+}\left(x_{i}\right), 1-\mu_{\tilde{A}}^{-}\left(x_{i}\right)-v_{\tilde{A}}^{-}\left(x_{i}\right)\right]$ and $i=1,2, \ldots, n$.

Xu [9] investigated methods for aggregating interval-valued intuitionistic fuzzy information and defined some related concepts of IVIFN as follows

Definition 2.2. [9] A pair $\tilde{\alpha}=\left(\mu_{\tilde{A}}\left(x_{i}\right), v_{\tilde{A}}\left(x_{i}\right)\right)$ is called by an IVIFN, denoted by $\tilde{\alpha}=$ 
$([a, b],[c, d])$, where $[a, b] \subseteq[0,1],[c, d] \subseteq[0,1]$ and $b+d \leq 1$

Let $\tilde{\alpha}=([a, b],[c, d])$ be an IVIFN and let $\lambda$ be a positive constant. Then the multiplication and exponential operations as follows

$$
\begin{aligned}
& \lambda \tilde{\alpha}=\left([1-(1-a), 1-(1-b)],\left[(c)^{\lambda},(d)^{\lambda}\right]\right) \\
& \tilde{\alpha}^{\lambda}=\left(\left[(a)^{\lambda},(b)^{\lambda}\right],[1-(1-c), 1-(1-d)]\right)
\end{aligned}
$$

Definition 2.3. [9] Let $\tilde{\alpha}_{1}, \tilde{\alpha}_{2}, \ldots, \tilde{\alpha}_{n}$ be n IVIFNs and let $\lambda_{j}$ be a positive constant as the weight of $\tilde{\alpha}_{j}$, where $\lambda_{j} \in[0,1], j=1,2, \ldots, n, \sum_{j=1}^{n} \lambda_{j}=1$. The interval-valued intuitionistic fuzzy weighted averaging (IVIFWA) operator is defined by

$\operatorname{IVIFW} A_{\lambda}\left(\tilde{\alpha}_{1}, \tilde{\alpha}_{2}, \ldots, \tilde{\alpha}_{n}\right)=\left(\left[1-\prod_{j=1}^{n}(1-a)^{\lambda_{j}}, 1-\prod_{j=1}^{n}(1-b)^{\lambda_{j}}\right],\left[\prod_{j=1}^{n}(c)^{\lambda_{j}}, \prod_{j=1}^{n}(d)^{\lambda_{j}}\right]\right)$

Definition 2.4. [9] Let $\tilde{\alpha}=([a, b],[c, d])$ be an IVIFN, score function $S_{X u}(\tilde{\alpha})$ and accuracy function $H_{X u}(\tilde{\alpha})$ of $\tilde{\alpha}$ is respectively defined as follows:

$$
\begin{aligned}
& S_{X u}(\tilde{\alpha})=\frac{a-c+b-d}{2} \\
& H_{X u}(\tilde{\alpha})=\frac{a+b+c+d}{2}
\end{aligned}
$$

where $S_{X u}(\tilde{\alpha}) \in[-1,1], H_{X u}(\tilde{\alpha}) \in[0,1]$. The larger the value of $S_{X u}(\tilde{\alpha})$, the larger the IVIFN $\tilde{\alpha}$.

Definition 2.5. [9] Let $\tilde{\alpha}_{1}=\left(\left[a_{1}, b_{1}\right],\left[c_{1}, d_{1}\right]\right)$ and $\tilde{\alpha}_{2}=\left(\left[a_{2}, b_{2}\right],\left[c_{2}, d_{2}\right]\right)$ be any two IVIFNs. Then

(1) If $S_{X u}\left(\tilde{\alpha}_{1}\right)>S_{X u}\left(\tilde{\alpha}_{2}\right)$, then $\tilde{\alpha}_{1}>\tilde{\alpha}_{2}$

(2) If $S_{X u}\left(\tilde{\alpha}_{1}\right)<S_{X u}\left(\tilde{\alpha}_{2}\right)$, then $\tilde{\alpha}_{1}<\tilde{\alpha}_{2}$

(3) If $S_{X u}\left(\tilde{\alpha}_{1}\right)=S_{X u}\left(\tilde{\alpha}_{2}\right)$, then 1) If $H_{X u}\left(\tilde{\alpha}_{1}\right)>H_{X u}\left(\tilde{\alpha}_{2}\right)$, then $\tilde{\alpha}_{1}>\tilde{\alpha}_{2}$ 
2) If $H_{X u}\left(\tilde{\alpha}_{1}\right)<H_{X u}\left(\tilde{\alpha}_{2}\right)$, then $\tilde{\alpha}_{1}<\tilde{\alpha}_{2}$

3) If $H_{X u}\left(\tilde{\alpha}_{1}\right)=H_{X u}\left(\tilde{\alpha}_{2}\right)$, then $\tilde{\alpha}_{1}=\tilde{\alpha}_{2}$

Example 2.1. Let $\tilde{\alpha}_{1}=([0.2,0.3],[0.4,0.5])$ and $\tilde{\alpha}_{2}=([0.1,0.4],[0.4,0.5])$ be two IVIFNs. According to Eqs. (4) and (5), we perceive that $S_{X u}\left(\tilde{\alpha}_{1}\right)=S_{X u}\left(\tilde{\alpha}_{2}\right)=-0.2$ and $H_{X u}\left(\tilde{\alpha}_{1}\right)=H_{X u}\left(\tilde{\alpha}_{2}\right)=0.7$. In this case, it can't distinguish which IVIFN is greater by Definition 2.5.

To overcome the shortcoming that Xu's score and accuracy function cannot distinguish the difference between two distinct IVIFNs in some cases, Wang [10] introduced the membership uncertainty (MU) index and the hesitation uncertainty (HU) index.

Definition 2.6. [10] Let $\tilde{\alpha}=([a, b],[c, d])$ be an IVIFN. The $M U$ index $M U(\tilde{\alpha})$ of $\tilde{\alpha}$ is defined as follows:

$$
M U(\tilde{\alpha})=b+c-a-d
$$

where $M U(\tilde{\alpha}) \in[-1,1]$. The larger the value of $M U(\tilde{\alpha})$, the smaller the IVIFN $\tilde{\alpha}$. The $H U$ index $H U(\tilde{\alpha})$ of the IVIFN $\tilde{\alpha}$ is defined as follows:

$$
H U(\tilde{\alpha})=b+d-a-c
$$

where $H U(\tilde{\alpha}) \in[0,1]$. The larger the value of $H U(\tilde{\alpha})$, the smaller the IVIFN $\tilde{\alpha}$.

Introducing the $M U$ index and the $H U$ index, Wang [10] proposed a new sorting relationship as shown below.

Definition 2.7. [10] Let $\tilde{\alpha}_{1}=\left(\left[a_{1}, b_{1}\right],\left[c_{1}, d_{1}\right]\right)$ and $\tilde{\alpha}_{2}=\left(\left[a_{2}, b_{2}\right],\left[c_{2}, d_{2}\right]\right)$ be any two IVIFNs. Then

(1) If $S_{X u}\left(\tilde{\alpha}_{1}\right)>S_{X u}\left(\tilde{\alpha}_{2}\right)$, then $\tilde{\alpha}_{1}>\tilde{\alpha}_{2}$

(2) If $S_{X u}\left(\tilde{\alpha}_{1}\right)<S_{X u}\left(\tilde{\alpha}_{2}\right)$, then $\tilde{\alpha}_{1}<\tilde{\alpha}_{2}$

(3) If $S_{X u}\left(\tilde{\alpha}_{1}\right)=S_{X u}\left(\tilde{\alpha}_{2}\right)$, then

1) If $H_{X u}\left(\tilde{\alpha}_{1}\right)>H_{X u}\left(\tilde{\alpha}_{2}\right)$, then $\tilde{\alpha}_{1}>\tilde{\alpha}_{2}$

2) If $H_{X u}\left(\tilde{\alpha}_{1}\right)<H_{X u}\left(\tilde{\alpha}_{2}\right)$, then $\tilde{\alpha}_{1}<\tilde{\alpha}_{2}$

3) If $H_{X u}\left(\tilde{\alpha}_{1}\right)=H_{X u}\left(\tilde{\alpha}_{2}\right)$, then 


$$
\begin{aligned}
& \text { i. If } T_{W}\left(\tilde{\alpha}_{1}\right)>T_{W}\left(\tilde{\alpha}_{2}\right) \text {, then } \tilde{\alpha}_{1}<\tilde{\alpha}_{2} \\
& \text { ii. If } T_{W}\left(\tilde{\alpha}_{1}\right)<T_{W}\left(\tilde{\alpha}_{2}\right) \text {, then } \tilde{\alpha}_{1}>\tilde{\alpha}_{2} \\
& \text { iii. If } T_{W}\left(\tilde{\alpha}_{1}\right)=T_{W}\left(\tilde{\alpha}_{2}\right) \text {, then }
\end{aligned}
$$
a. If $G_{W}\left(\tilde{\alpha}_{1}\right)>G_{W}\left(\tilde{\alpha}_{2}\right)$, then $\tilde{\alpha}_{1}<\tilde{\alpha}_{2}$
b. If $G_{W}\left(\tilde{\alpha}_{1}\right)<G_{W}\left(\tilde{\alpha}_{2}\right)$, then $\tilde{\alpha}_{1}>\tilde{\alpha}_{2}$
c. If $G_{W}\left(\tilde{\alpha}_{1}\right)=G_{W}\left(\tilde{\alpha}_{2}\right)$, then $\tilde{\alpha}_{1}=\tilde{\alpha}_{2}$

Because of Xu's ranking method's flaws, many scholars have done a lot of research on the score function and the accuracy function. For example, to overcome the drawback of Xu's accuracy function, Ye [11] proposed an improved accuracy as follows:

Definition 2.8. [11] Let $\tilde{\alpha}=([a, b],[c, d])$ be an IVIFN, $H_{Y e}(\tilde{\alpha})$ is called the accuracy function of the $\tilde{\alpha}$ as:

$$
H_{Y e}(\tilde{\alpha})=a+b-1+\frac{c+d}{2}
$$

where $H_{Y e}(\tilde{\alpha}) \in[0,1]$. The larger the value of $H_{Y e}(\tilde{\alpha})$, the larger the IVIFN $\tilde{\alpha}$.

Example 2.2. Let $\tilde{\alpha}_{1}=([0.3,0.5],[0.2,0.4])$ and $\tilde{\alpha}_{2}=([0.35,0.45],[0.25,0.35])$ be two IVIFNs. Calculated by Eq. (8), we can observe that $H_{Y e}\left(\tilde{\alpha}_{1}\right)=H_{Y e}\left(\tilde{\alpha}_{2}\right)=0.1$. In this case, the order between $\tilde{\alpha}_{1}$ and $\tilde{\alpha}_{2}$ cannot be determined.

Due to Definition 2.4 and Definition 2.8 fail to rank the comparable interval-valued intuitionistic fuzzy values for two IVIFNs, Nayagam [12] introduced a novel accuracy function as follows:

Definition 2.9. [12] Let $\tilde{\alpha}=([a, b],[c, d])$ be an IVIFN, the accuracy function $H_{V}(\tilde{\alpha})$ of the IVIFN $\tilde{\alpha}$ is defined as follows:

$$
H_{V}(\tilde{\alpha})=\frac{a+b-d(1-b)-c(1-a)}{2}
$$

where $H_{V}(\tilde{\alpha}) \in[0,1]$. The larger the value of $H_{V}(\tilde{\alpha})$, the larger the IVIFN $\tilde{\alpha}$.

However, the proposed techniques for ranking IVIFNs using a score function, the scope of solutions is limited. For this reason, Yao [13] proposed a new score function to overcome this 
problem.

Definition 2.10. [13] Let $\tilde{\alpha}=([a, b],[c, d])$ be an IVIFN, $S_{\text {Yao }}(\tilde{\alpha})$ is called the score function of the $\tilde{\alpha}$ as follows:

$$
S_{\text {Yao }}(\tilde{\alpha})=a+b-a b-\frac{a d+b c}{2}
$$

where $S_{\text {Yao }}(\tilde{\alpha}) \in[-1,1]$. The larger the value of $S_{\text {Yao }}(\tilde{\alpha})$, the larger the IVIFN $\tilde{\alpha}$.

Example 2.3. Let $\tilde{\alpha}_{1}=([0.35,0.45],[0.25,0.35])$ and $\tilde{\alpha}_{2}=([0.3,0.5],[0.2,0.4])$ be two IVIFNs. According to Eqs. (4)-(7), we can get that $S_{X u}\left(\tilde{\alpha}_{1}\right)=S_{X u}\left(\tilde{\alpha}_{2}\right)=0.1, H_{X u}\left(\tilde{\alpha}_{1}\right)=$ $H_{X u}\left(\tilde{\alpha}_{2}\right)=0.7, M U\left(\tilde{\alpha}_{1}\right)=M U\left(\tilde{\alpha}_{2}\right)=0, H U\left(\tilde{\alpha}_{2}\right)=0.2<H U\left(\tilde{\alpha}_{1}\right)=0.4$. So we have $\tilde{\alpha}_{1}>\tilde{\alpha}_{2}$. However, based on Eqs. (9)-(10), we obtain $\tilde{\alpha}_{1}<\tilde{\alpha}_{2}$ which is illogical.

Xu's score and accuracy function are only based on the membership and non-membership intervals of the IVIFNs. Considering the information of the hesitation interval of the IVIFNs, Wang [14] proposed a new score function as follows:

Definition 2.11. [14] Let $\tilde{\alpha}=([a, b],[c, d])$ be an IVIFN, the score function $S_{W}(\tilde{\alpha})$ of the IVIFN $\tilde{\alpha}$ is defined as follows:

$$
S_{W}(\tilde{\alpha})=\left(\frac{a+b}{2}-\frac{c+d}{2}\right)\left(1+\frac{(1-b-d)+(1-a-c)}{2}\right)
$$

where $S_{W}(\tilde{\alpha}) \in[-1,1]$. The larger the value of $S_{W}(\tilde{\alpha})$, the larger the IVIFN $\tilde{\alpha}$.

Example 2.4. Let $\tilde{\alpha}_{1}=([0.1,0.1],[0.1,0.1])$ and $\tilde{\alpha}_{2}=([0.3,0.3],[0.3,0.3])$ be two IVIFNs. From Eq. (11), we derived that $S\left(\tilde{\alpha}_{1}\right)=S\left(\tilde{\alpha}_{2}\right)=0$. In this case, we can't determine which IVIFN is greater.

Gao [15] proposed a decision analysis method based on prospect theory, which depends on a new score function to convert IVIFNs into real numbers. The proposed score function is shown as:

Definition 2.12. [15] Let $\tilde{\alpha}=([a, b],[c, d])$ be an IVIFN, $S_{G}(\tilde{\alpha})$ of the $\tilde{\alpha}$ is described as follows: 


$$
S_{G J W}(\tilde{\alpha})=\frac{a+b-c-d}{4-(a+b+c+d)}
$$

where $S_{G J W}(\tilde{\alpha}) \in[-1,1]$. The larger the value of $S_{G J W}(\tilde{\alpha})$, the larger the IVIFN $\tilde{\alpha}$.

Example 2.5. Let $\tilde{\alpha}_{1}=([0.2,0.4],[0.1,0.3])$ and $\tilde{\alpha}_{2}=([0.25,0.35],[0.15,0.25])$ be two IVIFNs. Using Eq. (12), we detect that $S_{G J W}\left(\tilde{\alpha}_{1}\right)=S_{G J W}\left(\tilde{\alpha}_{2}\right)=0.0667$. In this case, we can't distinguish which IVIFN is greater.

An improved interval intuitionistic fuzzy entropy and a new score function are proposed by Gao [16] for the multi-attribute decision making problem where the decision information is characterized by interval intuitionistic fuzzy number and attribute weights are not known in advance.

Definition 2.13. [16] Let $\tilde{\alpha}=([a, b],[c, d])$ be an IVIFN, the score function $S_{G M M}(\tilde{\alpha})$ of the $\tilde{\alpha}$ is denoted as follows:

$$
S_{G M M}(\tilde{\alpha})=\frac{1}{4}(a-c+b-d)\left(1+\frac{1}{a+b-a c+b d}\right)
$$

where $S_{G M M}(\tilde{\alpha}) \in[-1,1]$. The larger the value of $S_{G M M}(\tilde{\alpha})$, the larger the IVIFN $\tilde{\alpha}$.

Example 2.6. Let $\tilde{\alpha}_{1}=([0.2,0.2],[0.2,0.2])$ and $\tilde{\alpha}_{2}=([0.6,0.6],[0.6,0.6])$ be two IVIFNs. Based on Eq. (13), we detect that $S_{G M M}\left(\tilde{\alpha}_{1}\right)=S_{G M M}\left(\tilde{\alpha}_{2}\right)=0$. In this case, We can't figure out the correct order between $\tilde{\alpha}_{1}$ and $\tilde{\alpha}_{2}$.

Gong [18] analyzed the shortcomings of several current score functions and accuracy functions. To overcome these defects, Gong proposed original score functions and accuracy functions using the idea of probability theory's total probability formula.

Definition 2.14. [18] Let $\tilde{\alpha}=([a, b],[c, d])$ be an IVIFN, $S_{G R C}(\tilde{\alpha})$ is regarded the score function of the $\tilde{\alpha}$ is expressed as follows:

$$
S_{G R C}(\tilde{\alpha})=\frac{(d-c)^{2}-(b-a)^{2}+2(a+b)}{2(a+b+c+d)}
$$

where $S_{G R C}(\tilde{\alpha}) \in[-1,1]$. The larger the value of $S_{G R C}(\tilde{\alpha})$, the larger the IVIFN $\tilde{\alpha}$. 
Example 2.7. Let $\tilde{\alpha}_{1}=([0.25,0.45],[0.15,0.35])$ and $\tilde{\alpha}_{2}=([0.3,0.4],[0.2,0.3])$ be two IVIFNs. By Eq. (14), we discover that $S_{G R C}\left(\tilde{\alpha}_{1}\right)=S_{G R C}\left(\tilde{\alpha}_{2}\right)=0.5833$. In this case, we can't identify which IVIFN is greater.

Wang and Chen [17] proposed a new score function $S_{W C}$ of IVIFVs to develop an improved MADM method to overcome the drawback of Chen and Huang's MADM method.

Definition 2.15. [17] Let $\tilde{\alpha}=([a, b],[c, d])$ be an IVIFN, the proposed score function $S_{W C}(\tilde{\alpha})$ of the $\tilde{\alpha}$ as

$$
S_{W C}(\tilde{\alpha})=\frac{a+b+\sqrt{b d}(1-a-c)+\sqrt{a c}(1-b-d)}{2}
$$

where $S_{W C}(\tilde{\alpha}) \in[-1,1]$. The larger the value of $S_{W C}(\tilde{\alpha})$, the larger the IVIFN $\tilde{\alpha}$.

Example 2.8. Let $\tilde{\alpha}_{1}=([0,0.5],[0,0.5])$ and $\tilde{\alpha}_{2}=\left([0,0.6],\left[0, \frac{4}{15}\right]\right)$ be two IVIFNs. According to Eqs. (15), we notice that $S_{W C}\left(\tilde{\alpha}_{1}\right)=S_{W C}\left(\tilde{\alpha}_{2}\right)=0.5$. In this case, we can't distinguish which IVIFN is greater.

In general, the score function is a measure that characterizes the degree to which a decision object has a certain attribute. The higher the score value, the more the decision object has this characteristic; on the contrary, the less it has this attribute characteristic. And the accuracy function is a measure to evaluate whether an IVIFN accurately describes the attribute characteristics of the decision object. The larger the value of the accuracy function, the more accuracy the IVIFN is described; otherwise, the fuzzier the description is.

The main drawback of the above method is that they only focuses on the information of membership and non-membership of the IVIFN, but lacks consideration or insufficient use of the information about hesitation. In order to overcome the flaws of all the above methods, by comprehensively considering the information of IVIFN's membership, non-membership and hesitation, a more effective score function and accuracy function is proposed to rank IVIFNs as follows:

Definition 2.16. Let $\tilde{\alpha}=([a, b],[c, d])$ be an IVIFN. The proposed score function $S(\tilde{\alpha})$ of $\tilde{\alpha}$ is defined as follows:

$$
S(\tilde{\alpha})=\frac{a+b-\frac{\sqrt{a c}}{\sqrt{a c}+\sqrt{b d}}(1-b-d)-\frac{\sqrt{b d}}{\sqrt{a c}+\sqrt{b d}}(1-a-c)-c-d}{2}
$$


where $S(\tilde{\alpha}) \in[-1,1]$ and $\frac{0}{0}=1$. The larger the value of $S(\tilde{\alpha})$, the larger the IVIFN $\tilde{\alpha}$.

Definition 2.17. Let $\tilde{\alpha}=([a, b],[c, d])$ be an IVIFN. The proposed accuracy function $A(\alpha)$ of the IVIFN $\tilde{\alpha}$ is defined as follows:

$$
A(\alpha)=\frac{a+b+\frac{\sqrt{a c}}{\sqrt{a c}+\sqrt{b d}}(1-a-c)+\frac{\sqrt{b d}}{\sqrt{a c}+\sqrt{b d}}(1-b-d)}{2}
$$

where $A(\tilde{\alpha}) \in[0,1]$ and $\frac{0}{0}=1$. The larger the value of $A(\tilde{\alpha})$, the larger the IVIFN $\tilde{\alpha}$.

Definition 2.18. Let $\tilde{\alpha}_{1}=\left(\left[a_{1}, b_{1}\right],\left[c_{1}, d_{1}\right]\right)$ and $\tilde{\alpha}_{2}=\left(\left[a_{2}, b_{2}\right],\left[c_{2}, d_{2}\right]\right)$ be any two IVIFNs. Then

(1) If $S\left(\tilde{\alpha}_{1}\right)>S\left(\tilde{\alpha}_{2}\right)$, then $\tilde{\alpha}_{1}>\tilde{\alpha}_{2}$

(2) If $S\left(\tilde{\alpha}_{1}\right)<S\left(\tilde{\alpha}_{2}\right)$, then $\tilde{\alpha}_{1}<\tilde{\alpha}_{2}$

(3) If $S\left(\tilde{\alpha}_{1}\right)=S\left(\tilde{\alpha}_{2}\right)$, then

1) If $A\left(\tilde{\alpha}_{1}\right)>A\left(\tilde{\alpha}_{2}\right)$, then $\tilde{\alpha}_{1}>\tilde{\alpha}_{2}$

2) If $A\left(\tilde{\alpha}_{1}\right)<A\left(\tilde{\alpha}_{2}\right)$, then $\tilde{\alpha}_{1}<\tilde{\alpha}_{2}$

3) If $A\left(\tilde{\alpha}_{1}\right)=A\left(\tilde{\alpha}_{2}\right)$, then $\tilde{\alpha}_{1}=\tilde{\alpha}_{2}$

From Table 1, we can find that the score and accuracy functions introduced above will get an unreasonable ranking of IVIFNs in some situations. But based on the score and accuracy function defined in this paper, we can obtain the right sort effectively.

Table 1 A comparison of the ranking orders of IVIFNs for different score or accuracy functions

\begin{tabular}{cccc}
\hline Researches & \multicolumn{1}{c}{ IVIFNs } & $\begin{array}{c}\text { score or accuracy } \\
\text { functions }\end{array}$ & $\begin{array}{c}\text { The proposed } \\
\text { score function }\end{array}$ \\
\hline$[9]$ & $\tilde{\alpha}_{1}=([0.20,0.30],[0.40,0.50])$ & $S_{X u}\left(\tilde{\alpha}_{1}\right)=S_{X u}\left(\tilde{\alpha}_{2}\right)$ & $S\left(\tilde{\alpha}_{1}\right)>S\left(\tilde{\alpha}_{2}\right)$ \\
& $\tilde{\alpha}_{2}=([0.10,0.40],[0.40,0.50])$ & $H_{X u}\left(\tilde{\alpha}_{1}\right)=H_{X u}\left(\tilde{\alpha}_{2}\right)$ & \\
& $\tilde{\alpha}_{1}=([0.30,0.50],[0.20,0.40])$ & & \\
{$[11]$} & $\tilde{\alpha}_{2}=([0.35,0.45],[0.25,0.35])$ & $H_{Y e}\left(\tilde{\alpha}_{1}\right)=H_{Y e}\left(\tilde{\alpha}_{2}\right)$ & $S\left(\tilde{\alpha}_{1}\right)<S\left(\tilde{\alpha}_{2}\right)$ \\
& $\tilde{\alpha}_{1}=([0.35,0.45],[0.25,0.35])$ & & \\
{$[12]$} & $\tilde{\alpha}_{2}=([0.30,0.50],[0.20,0.40])$ & $H_{V}\left(\tilde{\alpha}_{1}\right)<H_{V}\left(\tilde{\alpha}_{2}\right)$ & $S\left(\tilde{\alpha}_{1}\right)>S\left(\tilde{\alpha}_{2}\right)$ \\
\hline
\end{tabular}


[13] $\quad \tilde{\alpha}_{1}=([0.35,0.45],[0.25,0.35])$

$$
S_{\text {Yао }}\left(\tilde{\alpha}_{1}\right)<S_{\text {Yао }}\left(\tilde{\alpha}_{2}\right) \quad S\left(\tilde{\alpha}_{1}\right)>S\left(\tilde{\alpha}_{2}\right)
$$$$
\tilde{\alpha}_{2}=([0.30,0.50],[0.20,0.40])
$$

$\tilde{\alpha}_{1}=([0.10,0.10],[0.10,0.10])$

$$
\tilde{\alpha}_{2}=([0.30,0.30],[0.30,0.30])
$$

$$
S_{W}\left(\tilde{\alpha}_{1}\right)=S_{W}\left(\tilde{\alpha}_{2}\right) \quad S\left(\tilde{\alpha}_{1}\right)<S\left(\tilde{\alpha}_{2}\right)
$$

$\tilde{\alpha}_{1}=([0.20,0.40],[0.10,0.30])$

$\tilde{\alpha}_{2}=([0.25,0.35],[0.15,0.25])$

$$
S_{G J W}\left(\tilde{\alpha}_{1}\right)=S_{G J W}\left(\tilde{\alpha}_{2}\right) \quad S\left(\tilde{\alpha}_{1}\right)<S\left(\tilde{\alpha}_{2}\right)
$$

$\tilde{\alpha}_{1}=([0.20,0.20],[0.20,0.20])$

[16] $\tilde{\alpha}_{2}=([0.60,0.60],[0.60,0.60])$

$$
S_{G M M}\left(\tilde{\alpha}_{1}\right)=S_{G M M}\left(\tilde{\alpha}_{2}\right) \quad S\left(\tilde{\alpha}_{1}\right)<S\left(\tilde{\alpha}_{2}\right)
$$

$\tilde{\alpha}_{1}=([0.00,0.50],[0.00,0.50])$

$\tilde{\alpha}_{2}=([0.00,0.60],[0.00,4 / 15])$

$$
S_{W C}\left(\tilde{\alpha}_{1}\right)=S_{W C}\left(\tilde{\alpha}_{2}\right) \quad S\left(\tilde{\alpha}_{1}\right)<S\left(\tilde{\alpha}_{2}\right)
$$

[18]

$$
\tilde{\alpha}_{1}=([0.25,0.45],[0.15,0.35])
$$

$$
\tilde{\alpha}_{2}=([0.30,0.40],[0.20,0.30])
$$

$$
S_{G R C}\left(\tilde{\alpha}_{1}\right)=S_{G R C}\left(\tilde{\alpha}_{2}\right) \quad S\left(\tilde{\alpha}_{1}\right)<S\left(\tilde{\alpha}_{2}\right)
$$

\section{Analyzing the flaws of Yue's MADM method}

\subsection{A review of Yue's MADM method}

In this section, we analyze the flaws of Yue's MADM method [27]. Let $A=\left\{A_{1}, A_{2}, \ldots, A_{m}\right\}$ be a set of alternatives, $U=\left\{u_{1}, u_{2}, \ldots, u_{n}\right\}$ be a set of attributes, $D=\left\{d_{1}, d_{2}, \ldots, d_{t}\right\}$ be a set of decision makers, and $W=\left\{w_{1}, w_{2}, \ldots, w_{n}\right\}$ be the weight vector of attributes, where $0 \leq w_{j} \leq 1, \quad \sum_{j=1}^{n} w_{j}=1$. We briefly review Yue's MADM method [27] as follows:

\section{Step 1. Establish group decision matrices}

For each alternative, the multiple attribute group decision making problem can be represented by the group decision matrix as follows:

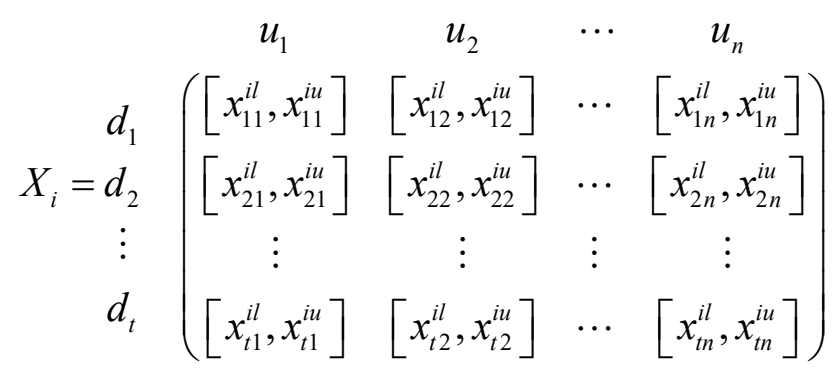

where $\left[x_{k j}^{i l}, x_{k j}^{i u}\right]$ is the interval value of attribute $u_{j}$ evaluates by decision maker $d_{k}$ 
corresponding to alternative $A_{i}, k=1,2, \ldots, t, j=1,2, \ldots, n, i=1,2, \ldots, m$.

Step 2. Normalize the decision matrix $X_{i}=\left[x_{k j}^{i l}, x_{k j}^{i u}\right]$ into the normalized matrix $Y_{i}=$ $\left[y_{k j}^{i l}, y_{k j}^{i u}\right]$

In general, there are benefit attributes and cost attributes in the multiple attribute decisionmaking problems [28]. Yue [27] introduced the new formulas to normalize each attribute value in $X_{i}$. The normalized group decision matrix $Y_{i}$ is constructed by Eq. (19) or (20) as follows [27]:

For benefit attribute:

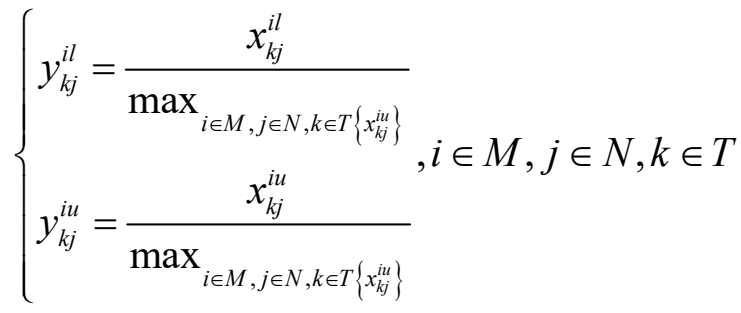

For cost attribute:

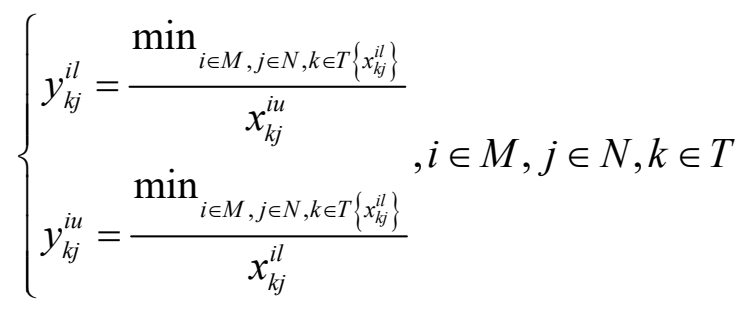

According to the above formulas, each attribute value $\left[x_{k j}^{i l}, x_{k j}^{i u}\right]$ in decision matrix $X_{i}$ can be normalized into $\left[y_{k j}^{i l}, y_{k j}^{i u}\right]$ in $Y_{i}$ as follows:

$$
\left.\left.\begin{array}{ccccc} 
& u_{1} & u_{2} & \cdots & u_{n} \\
Y_{i}=d_{2} & \left(\left[y_{11}^{i l}, y_{11}^{i u}\right]\right. & {\left[y_{12}^{i l}, y_{12}^{i u}\right]} & \cdots & {\left[y_{1 n}^{i l}, y_{1 n}^{i u}\right]} \\
\vdots & {\left[y_{21}^{i l}, x_{21}^{i u}\right]} & {\left[y_{22}^{i l}, y_{22}^{i u}\right]} & \cdots & {\left[y_{2 n}^{i l}, y_{2 n}^{i u}\right]} \\
\vdots & \vdots & \vdots & \vdots \\
d_{t} & {\left[y_{t 1}^{i l}, y_{t 1}^{i u}\right]} & {\left[y_{t 2}^{i l}, y_{t 2}^{i u}\right]} & \cdots & {\left[y_{t n}^{i l}, y_{t n}^{i u}\right.}
\end{array}\right]\right)
$$

Step 3. Calculate the mean values and standard deviations of endpoints of intervals in $Y_{i}$

In order to aggregate attribute vectors into an IVIFN, Yue introduced the idea of mean and variance in statistics. Yue pointed out that the average value and standard deviation of the left endpoints (or right endpoints) of the interval can reflect the degree of dissatisfaction (or satisfaction) to a certain extent. Therefore, the following formulas are proposed: 


$$
\begin{aligned}
& \bar{y}_{j}^{i l}=\frac{1}{t} \sum_{k=1}^{t} \bar{y}_{k j}^{i l}, \sigma_{j}^{i l}=\sqrt{\frac{1}{t-1} \sum_{k=1}^{t}\left(\bar{y}_{k j}^{i l}-\bar{y}_{j}^{i l}\right)^{2}}, i \in M, j \in N \\
& \bar{y}_{j}^{i u}=\frac{1}{t} \sum_{k=1}^{t} \bar{y}_{k j}^{i u}, \sigma_{j}^{i u}=\sqrt{\frac{1}{t-1} \sum_{k=1}^{t}\left(\bar{y}_{k j}^{i u}-\bar{y}_{j}^{i u}\right)^{2}}, i \in M, j \in N
\end{aligned}
$$

where $\bar{y}_{j}^{i l}$ and $\bar{y}_{j}^{i l}$ are called the mean values of endpoints of intervals of $Y_{j}^{i}$ in Eq. (21), the $\sigma_{j}^{i l}$ and $\sigma_{j}^{i u}$ are called the standard deviations of endpoints of intervals of $Y_{j}^{i}$.

Step 4. Determine the lower and upper bounds of the dissatisfaction interval and the lower and upper bounds of the satisfactory interval.

Considering that " 0.5 " is the natural boundary of $[0,1]$, the boundary of satisfaction interval and dissatisfaction interval are as follows

$$
\begin{aligned}
& \xi_{i j}^{l}=\max \left\{\bar{y}_{j}^{i u}-\sigma_{j}^{i u}, 0.5\right\}, \quad \xi_{i j}^{u}=\bar{y}_{j}^{i u}+\sigma_{j}^{i u}, i \in M, j \in N \\
& \zeta_{i j}^{l}=\bar{y}_{j}^{i l}-\sigma_{j}^{i l}, \quad \zeta_{i j}^{u}=\min \left\{\bar{y}_{j}^{i l}+\sigma_{j}^{i l}, 0.5\right\}, i \in M, j \in N
\end{aligned}
$$

where $\xi_{i j}^{l}$ and $\xi_{i j}^{u}$ are the lower and upper boundaries of the satisfaction interval, similarly, $\zeta_{i j}^{l}$ and $\zeta_{i j}^{u}$ are the lower and upper boundaries of the dissatisfaction interval.

Step 5. Perform linear transformation on the endpoints of the dissatisfaction interval and the satisfaction interval.

Reflecting that the larger the element in $\left[\xi_{i j}^{l}, \xi_{i j}^{u}\right]$, the larger the satisfactory degree. Yue makes a linear transformation for $\left[\xi_{i j}^{l}, \xi_{i j}^{u}\right]$ by using the formula as follow:

$$
\kappa_{i j}^{l}=2 \xi_{i j}^{l}-1, \kappa_{i j}^{u}=2 \xi_{i j}^{u}-1
$$

Correspondingly, making a linear transformation for $\left[\zeta_{i j}^{l}, \zeta_{i j}^{u}\right]$ by

$$
\varsigma_{i j}^{l}=1-2 \zeta_{i j}^{u}, \varsigma_{i j}^{u}=1-2 \zeta_{i j}^{l}
$$

Step 6. Calculate the induced IVIFN of $Y_{j}^{i}$, then obtain the collective decision matrix.

To preserve the $\left(\left[\kappa_{i j}^{l}, \kappa_{i j}^{u}\right]\left[\varsigma_{i j}^{l}, \varsigma_{i j}^{u}\right]\right)$ with the form of IVIFN in Definition 2.1 and the condition $\kappa_{i j}^{u}+\varsigma_{i j}^{u} \leq 1$, a linear transformation for $\kappa_{i j}^{l}, \kappa_{i j}^{u}, \varsigma_{i j}^{l}, \varsigma_{i j}^{u}$ is made by Yue [27] as follow: 


$$
\mu_{i j}^{l}=\frac{\kappa_{i j}^{l}}{\tau_{i j}}, \mu_{i j}^{u}=\frac{\kappa_{i j}^{u}}{\tau_{i j}}, v_{i j}^{l}=\frac{\varsigma_{i j}^{l}}{\tau_{i j}}, v_{i j}^{u}=\frac{\varsigma_{i j}^{u}}{\tau_{i j}}
$$

where $\tau_{i j}=\max _{i \in M} \max _{j \in N}\left\{\kappa_{i j}^{l}+\kappa_{i j}^{u}+\varsigma_{i j}^{l}+\varsigma_{i j}^{u}\right\}$

Step 7. Calculate the overall evaluation

According to Definition 2.3, all elements in each row of the matrix are aggregated to the overall IVIFN of the alternative $A_{i}$.

Step 8. Rank the preference order of the alternatives.

According to Xu's score and accuracy function shown in Definition 2.4 and the ranking method of IVIFVs shown in Definition 2.5, we obtain alternatives' the preference order.

However, in this paper, we point out that Yue's method [27] has the following shortcomings:

a. In Step 2 of the method, it may lose information, which may not distinguish the preference order of alternatives in some situations.

b. Some parameters of the alternatives obtained in Step 4 may be in reverse order, which is illogical.

c. The collective evaluation results of each attribute characterized by IVIFNs obtained in Step 6 may violate the relevant definition of IVIFN in Yue's method.

In the following, we use two counter-examples to illustrate the drawbacks of Yue's MADM method [27].

\subsection{The counter example of Yue's MADM method}

\subsubsection{The counter example of incorrect preference ranking}

Let $A=\left\{A_{1}, A_{2}\right\}$ is the set of the alternatives and $D=\left\{d_{1}, d_{2}, d_{3}, d_{4}\right\}$ is a group of decision makers. $w=\left\{w_{1}, w_{2}\right\}$ is the weights set of the attributes $A_{1}, A_{2}$, which are provided by decision makers and $U=\left\{u_{1}, u_{2}\right\}$ is a finite set of attributes. Decision makers evaluate the alternatives $A_{1}$ and $A_{2}$ with respect to attributes $u_{1}$ by using the hundred-mark system and attributes $u_{2}$ by using the sixty-point system and attributes. Therefore, the score values of attributes are shown in Table 2.

Table 2 Decision matrix $X_{i}$

\begin{tabular}{cccc}
\hline Decision matrices & DMs & $u_{1}$ & $u_{2}$ \\
\hline$X_{1}$ & $d_{1}$ & {$[70,100]$} & {$[50,60]$} \\
& $d_{2}$ & {$[30,35]$} & {$[40,50]$} \\
\hline
\end{tabular}




\begin{tabular}{cccc}
\hline & $d_{3}$ & {$[50,60]$} & {$[30,50]$} \\
$d_{4}$ & {$[40,50]$} & {$[40,60]$} \\
$X_{2}$ & {$[40,50]$} & {$[50,60]$} \\
& $d_{1}$ & {$[30,35]$} & {$[45,55]$} \\
& $d_{3}$ & {$[40,50]$} & {$[40,60]$} \\
& $d_{4}$ & {$[60,90]$} & {$[50,60]$} \\
\hline
\end{tabular}

Consider the case where the weight vector is $w=\left(w_{1}, w_{2}\right)=(0.5,0.5)$. It is apparent that $A_{2} \succ A_{1}$

Based on Eq. (19) and the decision matrix $X_{i}$, We can obtain the normalized matrix $Y_{i}$ shown in Table 3.

Table 3 Normalized decision matrix $Y_{i}$

\begin{tabular}{cccc}
\hline Decision matrices & DMs & $u_{1}$ & $u_{2}$ \\
\hline$Y_{1}$ & $d_{1}$ & {$[0.70,1.00]$} & {$[0.50,0.60]$} \\
& $d_{2}$ & {$[0.30,0.35]$} & {$[0.40,0.50]$} \\
& $d_{3}$ & {$[0.50,0.60]$} & {$[0.30,0.50]$} \\
& $d_{4}$ & {$[0.40,0.50]$} & {$[0.40,0.60]$} \\
$Y_{2}$ & $d_{1}$ & {$[0.40,0.50]$} & {$[0.50,0.60]$} \\
& $d_{2}$ & {$[0.30,0.35]$} & {$[0.45,0.55]$} \\
& $d_{3}$ & {$[0.40,0.50]$} & {$[0.40,0.60]$} \\
& $d_{4}$ & {$[0.60,0.90]$} & {$[0.50,0.60]$} \\
\hline
\end{tabular}

By Eqs. (22)-(27) and the normalized matrix $Y_{i}$ obtained in Steps 2-5, we can acquire the required parameters of each alternative $A_{i}$ respectively, which are shown in Table 4 and Table 5.

Table 4 The parameters of attributes of alternative $A_{1}$

\begin{tabular}{ccccccc}
\hline Attributes & Parameters & Values & Parameters & Values & Parameters & Values \\
\hline$u_{1}$ & $\bar{y}_{1}^{1 l}$ & 0.4750 & $\varsigma_{1}^{l l}$ & 0.0000 & $\xi_{11}^{l}$ & 0.5352 \\
& $\sigma_{1}^{1 l}$ & 0.0592 & $\varsigma_{1}^{1 u}$ & 0.1683 & $\xi_{11}^{u}$ & 0.6898 \\
\hline
\end{tabular}




\begin{tabular}{ccccccc}
\hline$\zeta_{11}^{l}$ & 0.4158 & $\bar{y}_{1}^{1 u}$ & 0.6125 & $\kappa_{11}^{l}$ & 0.0704 \\
$\bar{y}_{1}^{1 l}+\sigma_{1}^{1 l}$ & 0.5342 & $\sigma_{1}^{1 u}$ & 0.0773 & $\kappa_{11}^{u}$ & 0.3796 \\
$\zeta_{11}^{u}$ & 0.5000 & $\bar{y}_{1}^{1 u}-\sigma_{1}^{1 u}$ & 0.5352 & $\tau_{11}$ & 0.6183 \\
$\bar{y}_{2}^{1 l}$ & 0.4000 & $\varsigma_{2}^{1 l}$ & 0.1867 & $\xi_{12}^{l}$ & 0.5467 \\
$\sigma_{2}^{1 l}$ & 0.0067 & $\varsigma_{2}^{1 u}$ & 0.2133 & $\xi_{12}^{u}$ & 0.5533 \\
$\zeta_{12}^{l}$ & 0.3933 & $\bar{y}_{2}^{1 u}$ & 0.5500 & $\kappa_{12}^{l}$ & 0.0933 \\
$\bar{y}_{2}^{1 l}+\sigma_{2}^{1 l}$ & 0.4067 & $\sigma_{2}^{1 u}$ & 0.0033 & $\kappa_{12}^{u}$ & 0.1067 \\
$\zeta_{12}^{u}$ & 0.4067 & $\bar{y}_{2}^{1 u}-\sigma_{2}^{1 u}$ & 0.5467 & $\tau_{12}$ & 0.6000 \\
\hline
\end{tabular}

Table 5 The parameters of attributes of alternative $A_{2}$

\begin{tabular}{ccccccc}
\hline Attributes & Parameters & Values & Parameters & Values & Parameters & Values \\
\hline$u_{1}$ & $\bar{y}_{1}^{2 l}$ & 0.4250 & $\varsigma_{1}^{2 l}$ & 0.1183 & $\xi_{21}^{l}$ & 0.5069 \\
& $\sigma_{1}^{2 l}$ & 0.0158 & $\varsigma_{1}^{2 u}$ & 0.1817 & $\xi_{21}^{u}$ & 0.6181 \\
$\zeta_{21}^{l}$ & 0.4092 & $\bar{y}_{1}^{2 u}$ & 0.5625 & $\kappa_{21}^{l}$ & 0.0137 \\
& $\bar{y}_{1}^{2 l}+\sigma_{1}^{2 l}$ & 0.4408 & $\sigma_{1}^{2 u}$ & 0.0556 & $\kappa_{21}^{u}$ & 0.2363 \\
& $\zeta_{21}^{u}$ & 0.4408 & $\bar{y}_{1}^{2 u}-\sigma_{1}^{2 u}$ & 0.5069 & $\tau_{21}$ & 0.5500 \\
$u_{2}$ & $\bar{y}_{2}^{2 l}$ & 0.4625 & $\varsigma_{2}^{2 l}$ & 0.0704 & $\xi_{22}^{l}$ & 0.5860 \\
& $\sigma_{2}^{2 l}$ & 0.0023 & $\varsigma_{2}^{2 u}$ & 0.0796 & $\xi_{22}^{u}$ & 0.5890 \\
& $\zeta_{22}^{l}$ & 0.4602 & $\bar{y}_{2}^{2 u}$ & 0.5875 & $\kappa_{22}^{l}$ & 0.1721 \\
& $\bar{y}_{2}^{2 l}+\sigma_{2}^{2 l}$ & 0.4648 & $\sigma_{2}^{2 u}$ & 0.0015 & $\kappa_{22}^{u}$ & 0.1779 \\
& $\zeta_{22}^{u}$ & 0.4648 & $\bar{y}_{2}^{2 u}-\sigma_{2}^{2 u}$ & 0.5860 & $\tau_{22}$ & 0.5000 \\
\hline
\end{tabular}

To compare the results, as we supposed at the beginning, we set each attribute's weight to be $w_{1}=w_{2}=0.5$. Using Eq. (28), we can obtain the collective evaluation of each attribute's results characterized by IVIFNs in Table 6. The score of the overall evaluation and the preference order of each attribute can be calculated by Definition 2.4 and Definition 2.5, which are summarized in Table 7. 


\begin{tabular}{cccc}
\hline Alternatives & $u_{1}$ & $u_{2}$ \\
\hline$A_{1}$ & $([0.1139,0.6139],[0.0000,0.2722])$ & $([0.1509,0.1726],[0.3020,0.3450])$ \\
$A_{2}$ & $([0.0222,0.3822],[0.1913,0.2939])$ & $([0.2783,0.2877],[0.1913,0.2939])$ \\
\hline \multicolumn{2}{c}{ Table 7 The score of the overall evaluation and the preference order of each alternatives $A_{i}$} \\
\hline Alternatives & overall evaluation & Scores & Ranking \\
\hline$A_{1}$ & $([0.1326,0.4348],[0.0000,0.3064])$ & 0.1305 & 1 \\
$A_{2}$ & $([0.1600,0.3366],[0.1913,0.2939])$ & 0.0057 & 2 \\
\hline
\end{tabular}

We can see that the preference order of alternatives is $A_{1} \succ A_{2}$, which is not consistent with the actual situation of $A_{1} \prec A_{2}$.

Therefore, it is obvious that Yue's method gets an incorrect order of $A_{1}$ and $A_{2}$. Yue's method has the flaw that it cannot get the correct preference order of alternatives due to the loss of information in some situations. The reason for this problem is that in the normalization process, Eqs. (19) and (20) only consider the distribution information of the data itself(local) but ignore the overall relationship with the attribute interval(global). Therefore, in the normalization process, we will introduce attribute interval information to overcome this defect.

3.2.2 The counter example of illogical parameters and faulty evaluation results

Let $A=\left\{A_{1}, A_{2}\right\}$ is the set of the alternatives and $D=\left\{d_{1}, d_{2}, d_{3}, d_{4}\right\}$ is a group of decision makers. $w=\left\{w_{1}, w_{2}\right\}$ is the weights set of the attributes $A_{1}, A_{2}$, which are provided by decision makers and $U=\left\{u_{1}, u_{2}\right\}$ is a finite set of attributes. Decision makers evaluate the alternatives $A_{1}, A_{2}$, with respect to attributes $u_{1}, u_{2}$ by using the hundred mark system. Therefore, the scores of attributes are shown in Table 8.

Table 8 Decision matrix $X_{i}$

\begin{tabular}{cccc}
\hline Decision matrices & DMs & $u_{1}$ & $u_{2}$ \\
\hline$X_{1}$ & $d_{1}$ & {$[70,80]$} & {$[8,10]$} \\
& $d_{2}$ & {$[80,85]$} & {$[9,10]$} \\
& $d_{3}$ & {$[90,100]$} & {$[8,9]$} \\
& $d_{4}$ & {$[70,75]$} & {$[9,10]$} \\
$X_{2}$ & $d_{1}$ & {$[65,80]$} & {$[8,9]$} \\
\hline
\end{tabular}




\begin{tabular}{ccc}
\hline$d_{2}$ & {$[75,85]$} & {$[1,2]$} \\
$d_{3}$ & {$[90,95]$} & {$[1,2]$} \\
$d_{4}$ & {$[65,75]$} & {$[1,3]$} \\
\hline
\end{tabular}

Let weight vector is $w=\left(w_{1}, w_{2}\right)=(0.5,0.5)$. It is apparent that $A_{1} \succ A_{2}$.

Based on Eq. (19) and the decision matrix $X_{i}$, We can obtain the normalized matrix shown in Table 9.

Table 9 Normalized decision matrix $Y_{i}$

\begin{tabular}{cccc}
\hline Decision matrices & DMs & $u_{1}$ & $u_{2}$ \\
\hline$Y_{1}$ & $d_{1}$ & {$[0.7,0.8]$} & {$[0.08,0.1]$} \\
& $d_{2}$ & {$[0.8,0.85]$} & {$[0.09,0.1]$} \\
& $d_{3}$ & {$[0.9,1]$} & {$[0.08,0.09]$} \\
& $d_{4}$ & {$[0.7,0.75]$} & {$[0.09,0.1]$} \\
$Y_{2}$ & $d_{1}$ & {$[0.65,0.8]$} & {$[0.08,0.09]$} \\
& $d_{2}$ & {$[0.75,0.85]$} & {$[0.01,0.02]$} \\
& $d_{3}$ & {$[0.9,0.95]$} & {$[0.01,0.02]$} \\
& $d_{4}$ & {$[0.65,0.75]$} & {$[0.01,0.03]$} \\
\hline
\end{tabular}

By Eqs. (22)-(27) and the normalized matrix $Y_{i}$ obtained in Steps 2-5, we can get the required parameters of each alternative $A_{i}$ respectively, which are shown in Table 10-11.

Table 10 The parameters of attributes of alternative $A_{1}$

\begin{tabular}{ccccccc}
\hline Attributes & Parameters & Values & Parameters & Values & Parameters & Values \\
\hline$u_{1}$ & $\bar{y}_{1}^{1 l}$ & 0.775 & $\varsigma_{1}^{1 l}$ & 0 & $\xi_{11}^{l}$ & 0.8383 \\
& $\sigma_{1}^{1 l}$ & 0.0092 & $\varsigma_{1}^{1 u}$ & -0.5317 & $\xi_{11}^{u}$ & 0.8617 \\
& $\zeta_{11}^{l}$ & 0.7658 & $\bar{y}_{1}^{1 u}$ & 0.85 & $\kappa_{11}^{l}$ & 0.6767 \\
& $\bar{y}_{1}^{1 l}+\sigma_{1}^{1 l}$ & 0.7842 & $\sigma_{1}^{1 u}$ & 0.0117 & $\kappa_{11}^{u}$ & 0.7233 \\
& $\zeta_{11}^{u}$ & 0.5 & $\bar{y}_{1}^{1 u}-\sigma_{1}^{1 u}$ & 0.8383 & $\tau_{11}$ & 0.8683 \\
$u_{2}$ & $\bar{y}_{2}^{1 l}$ & 0.0850 & $\varsigma_{2}^{1 l}$ & 0.8299 & $\xi_{12}^{l}$ & 0.5 \\
\hline
\end{tabular}




\begin{tabular}{cccccc}
\hline$\sigma_{2}^{1 l}$ & 0.00003 & $\varsigma_{2}^{1 u}$ & 0.8301 & $\xi_{12}^{u}$ & 0.097475 \\
$\zeta_{12}^{l}$ & 0.08497 & $\bar{y}_{2}^{1 u}$ & 0.0975 & $\kappa_{12}^{l}$ & 0 \\
$\bar{y}_{2}^{1 l}+\sigma_{2}^{1 l}$ & 0.08503 & $\sigma_{2}^{1 u}$ & 0.000025 & $\kappa_{12}^{u}$ & -0.8050 \\
$\zeta_{12}^{u}$ & 0.08503 & $\bar{y}_{2}^{1 u}-\sigma_{2}^{1 u}$ & 0.8302 & $\tau_{12}$ & 0.855 \\
\hline
\end{tabular}

Table 11 The parameters of attributes of alternative $A_{2}$

\begin{tabular}{ccccccc}
\hline Attributes & Parameters & Values & Parameters & Values & Parameters & Values \\
\hline$u_{1}$ & $\bar{y}_{1}^{2 l}$ & 0.7375 & $\varsigma_{1}^{2 l}$ & 0 & $\xi_{21}^{l}$ & 0.8302 \\
& $\sigma_{1}^{2 l}$ & 0.014 & $\varsigma_{1}^{2 u}$ & -0.4471 & $\xi_{21}^{u}$ & 0.8448 \\
$\zeta_{21}^{l}$ & 0.7235 & $\bar{y}_{1}^{2 u}$ & 0.8375 & $\kappa_{21}^{l}$ & 0.6604 \\
& $\bar{y}_{1}^{2 l}+\sigma_{1}^{2 l}$ & 0.7515 & $\sigma_{1}^{2 u}$ & 0.0073 & $\kappa_{21}^{u}$ & 0.6896 \\
& $\zeta_{21}^{u}$ & 0.5 & $\bar{y}_{1}^{2 u}-\sigma_{1}^{2 u}$ & 0.8302 & $\tau_{21}$ & 0.9029 \\
$u_{2}$ & $\bar{y}_{2}^{2 l}$ & 0.0275 & $\varsigma_{2}^{2 l}$ & 0.9426 & $\xi_{22}^{l}$ & 0.5 \\
& $\sigma_{2}^{2 l}$ & 0.0012 & $\varsigma_{2}^{2 u}$ & 0.9475 & $\xi_{22}^{u}$ & 0.0411 \\
& $\zeta_{22}^{l}$ & 0.0263 & $\bar{y}_{2}^{2 u}$ & 0.04 & $\kappa_{22}^{l}$ & 0 \\
& $\bar{y}_{2}^{2 l}+\sigma_{2}^{2 l}$ & 0.0287 & $\sigma_{2}^{2 u}$ & 0.0011 & $\kappa_{22}^{u}$ & -0.9177 \\
$\zeta_{22}^{u}$ & 0.0287 & $\bar{y}_{2}^{2 u}-\sigma_{2}^{2 u}$ & 0.0389 & $\tau_{22}$ & 0.9724 \\
\hline
\end{tabular}

To compare the results, as we supposed at the beginning, we set the weight of each attribute to be $w_{1}=w_{2}=0.5$. Using Eq. (28), we can obtain the collective evaluation of each attribute's results characterized by IVIFNs in the Table 10. The score of the overall evaluation and the preference order of each attribute can be calculated by Definition 2.4 and Definition 2.5, which are summarized in Table 12.

Table 12 The collective evaluation of each alternative $A_{i}$

\begin{tabular}{ccc}
\hline Alternatives & $u_{1}$ & $u_{2}$ \\
\hline$A_{1}$ & $([0.6959,0.7438],[0,-0.5468])$ & $([0,-0.8278],[0.8535,0.8537])$ \\
$A_{2}$ & $([0.6791,0.7092],[0,-0.4598])$ & $([0,-0.9437],[0.9694,0.9744])$ \\
\hline
\end{tabular}

From the data in Table 12, we can detect that Yue's method [27] has the drawbacks that it may 
get some illogical parameters of alternatives and some of them violate their own definition of IVIFN in this situation(the left endpoint of the interval is greater than the right endpoint, and the interval is less than 0 ). To overcome the above limitations, considering the characteristics of interval data and reducing the influence of outlier information through weights, a transformation matrix based on principal component analysis, set distribution weights, and a novel mapping strategy are introduced to propose a novel method for aggregating IVIFNs.

\section{The proposed MADM method based on the new score and accuracy function}

\subsection{Algorithm of the proposed MADM method}

In this section, we propose a novel MADM method to overcome the shortages of the Yue's method. Let $A=\left\{A_{1}, A_{2}, \ldots, A_{m}\right\}$ be a set of alternatives, $U=\left\{u_{1}, u_{2}, \ldots, u_{n}\right\}$ be a set of attributes, $D=\left\{d_{1}, d_{2}, \ldots, d_{t}\right\}$ be a set of decision makers, and $W=\left\{w_{1}, w_{2}, \ldots, w_{n}\right\}$ be the weight vector of attributes, where $0 \leq w_{j} \leq 1, \quad \sum_{j=1}^{n} w_{j}=1$. The improved MADM method shows as follows:

Step 1. Establish the decision matrix (DM) offered by the decision-maker can be described as Eq. (18).

Step 2. To address the loss of information in Yue's method, by extending the normalized method [23] to interval numbers, we establish the improved benefit attribute $U_{B}$ and cost attribute $U_{C}$, where $U=U_{B} \cup U_{C}$, as follows:

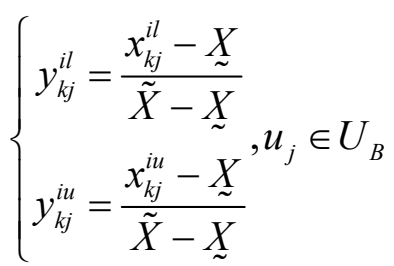

$$
\begin{aligned}
& \left\{\begin{array}{l}
y_{k j}^{i l}=\frac{\tilde{X}-x_{k j}^{i u}}{\tilde{X}-\underset{\sim}{X}} \\
y_{k j}^{i u}=\frac{\tilde{X}-x_{k j}^{i l}}{\tilde{X}-\underset{\sim}{X}}, u_{j} \in U_{C}
\end{array}\right.
\end{aligned}
$$

Considering the attributes $u_{j}(j=1,2, \ldots, n)$, the $\tilde{X}$ and $\underset{\sim}{X}$ are the maximum grade and the minimum grade of $u_{j}$, respectively. For example, the maximum grade and minimum grade are 100 and 0 respectively in the hundred-mark system, and it can be expressed as $\tilde{X}=100$ and $\underset{\sim}{X}=0$. 
Step 3. Based on the normalized matrix $Y_{i}=\left(\left[y_{k j}^{i l}, y_{k j}^{i u}\right]\right)_{t \times n}$, and the idea of Centers Principal Component Analysis(CPCA) [29], we build the transformed decision matrix $R$, where $R_{i j}=$ $\frac{y_{k j}^{i l}+y_{k j}^{i u}}{2}$

Step 4: Constructing the satisfaction set $R_{i j}^{s}$, dissatisfaction set $R_{i j}^{d}$ and uncertainty set $R_{i j}^{u}$ from the $j(1,2, \ldots, n)$ column of the transformed decision matrix $R$, shown as follow:

$$
\begin{aligned}
& R_{i j}^{s}=\left\{r_{k j}^{i} \mid r_{i j}^{i}>0.5, r_{k j}^{i} \in R_{i j}\right\} \\
& R_{i j}^{d}=\left\{r_{k j}^{i} \mid r_{i j}^{i}<0.5, r_{k j}^{i} \in R_{i j}\right\} \\
& R_{i j}^{u}=\left\{r_{k j}^{i} \mid r_{i j}^{i}=0.5, r_{k j}^{i} \in R_{i j}\right\}
\end{aligned}
$$

Step 5. Determine the bound(s) of the dissatisfaction interval and the satisfactory interval in $R_{i}$ by

$$
\begin{aligned}
& \xi_{i j}^{l}=\min _{k \in T}\left\{r_{k j}^{i} \mid r_{k j}^{i} \in R_{i j}^{s}\right\}, \xi_{i j}^{u}=\max _{k \in T}\left\{r_{k j}^{i} \mid r_{k j}^{i} \in R_{i j}^{s}\right\} \\
& \zeta_{i j}^{l}=\min _{k \in T}\left\{r_{k j}^{i} \mid r_{k j}^{i} \in R_{i j}^{d}\right\}, \zeta_{i j}^{u}=\max _{k \in T}\left\{r_{k j}^{i} \mid r_{k j}^{i} \in R_{i j}^{d}\right\}
\end{aligned}
$$

where $\xi_{i j}^{l}, \xi_{i j}^{u}, \zeta_{i j}^{l}$ and $\zeta_{i j}^{u}$ are called respectively, the lower and upper bounds of the dissatisfaction interval and the lower and upper bounds of the satisfactory interval.

Consider the case of the empty set, it can be analyzed in the following two cases:

Case I. If $R_{i j}^{s}=\varnothing$, then $\xi_{i j}^{l}=\xi_{i j}^{u}=0.5$.

Case II. If $R_{i j}^{d}=\varnothing$, then $\zeta_{i j}^{l}=\zeta_{i j}^{u}=0.5$.

Step 6. Based on Eqs. (26)-(27) and the bound(s) of the satisfactory and dissatisfactory interval obtained in Step 5, we can take a linear transformation for the endpoints of satisfaction and dissatisfaction interval.

Step 7. Calculating the group decision with the IVIFNs

Furthermore, to transform $\left[\xi_{i j}^{l}, \xi_{i j}^{u}\right]\left[\zeta_{i j}^{l}, \zeta_{i j}^{u}\right]$ into an IVIFN described in Definition 2.1, we make a linear transformation for parameters $\xi_{i j}^{l}, \xi_{i j}^{u}, \zeta_{i j}^{l}$, and $\zeta_{i j}^{u}$. To reduce the impact of individual data, considering the global distribution of the data, we introduce the proportions of the elements from the three sets in all elements as weights, as follows: 


$$
\begin{aligned}
& \mu_{i j}^{l}=\frac{\left|R_{i j}^{s}\right|}{\left|R_{i j}^{s}\right|+\left|R_{i j}^{d}\right|+\left|R_{i j}^{u}\right|} \frac{\kappa_{i j}^{l}}{\tau_{i j}}, \mu_{i j}^{u}=\frac{\left|R_{i j}^{s}\right|}{\left|R_{i j}^{s}\right|+\left|R_{i j}^{d}\right|+\left|R_{i j}^{u}\right|} \frac{\kappa_{i j}^{u}}{\tau_{i j}} \\
& v_{i j}^{l}=\frac{\left|R_{i j}^{d}\right|}{\left|R_{i j}^{s}\right|+\left|R_{i j}^{d}\right|+\left|R_{i j}^{u}\right|} \frac{\varsigma_{i j}^{l}}{\tau_{i j}}, v_{i j}^{u}=\frac{\left|R_{i j}^{d}\right|}{\left|R_{i j}^{s}\right|+\left|R_{i j}^{d}\right|+\left|R_{i j}^{u}\right|} \frac{\varsigma_{i j}^{u}}{\tau_{i j}}
\end{aligned}
$$

where $\tau_{i j}=\left(\kappa_{i j}^{l}+\kappa_{i j}^{u}+\varsigma_{i j}^{l}+\varsigma_{i j}^{u}+\frac{\left|R_{i j}^{u}\right|}{\left|R_{i j}^{s}\right|+\left|R_{i j}^{d}\right|+\left|R_{i j}^{u}\right|}\right), \frac{\left|R_{i j}^{u}\right|}{\left|R_{i j}^{s}\right|+\left|R_{i j}^{d}\right|+\left|R_{i j}^{u}\right|} \in[0,1]$. And $\left|R_{i j}^{s}\right|$, $\left|R_{i j}^{d}\right|,\left|R_{i j}^{u}\right|$ are the number of elements in the satisfactory set, dissatisfactory set and uncertain set, respectively. Moreover, considering that " 0.5 " is the natural boundary of $[0,1]$ and also represents the point of maximum hesitation before the linear change. We introduce the proportion of the elements of the hesitation set in all elements to reflect the hesitation information after the linear change.

Step 8. Based on Definition 2.3, calculate the overall evaluation for each alternative.

Step 9. Rank the preference order of the alternatives based on Definition 2.18 and overall evaluation, according to score function and accuracy function calculated by Eqs. (16)-(17) for each alternative. 


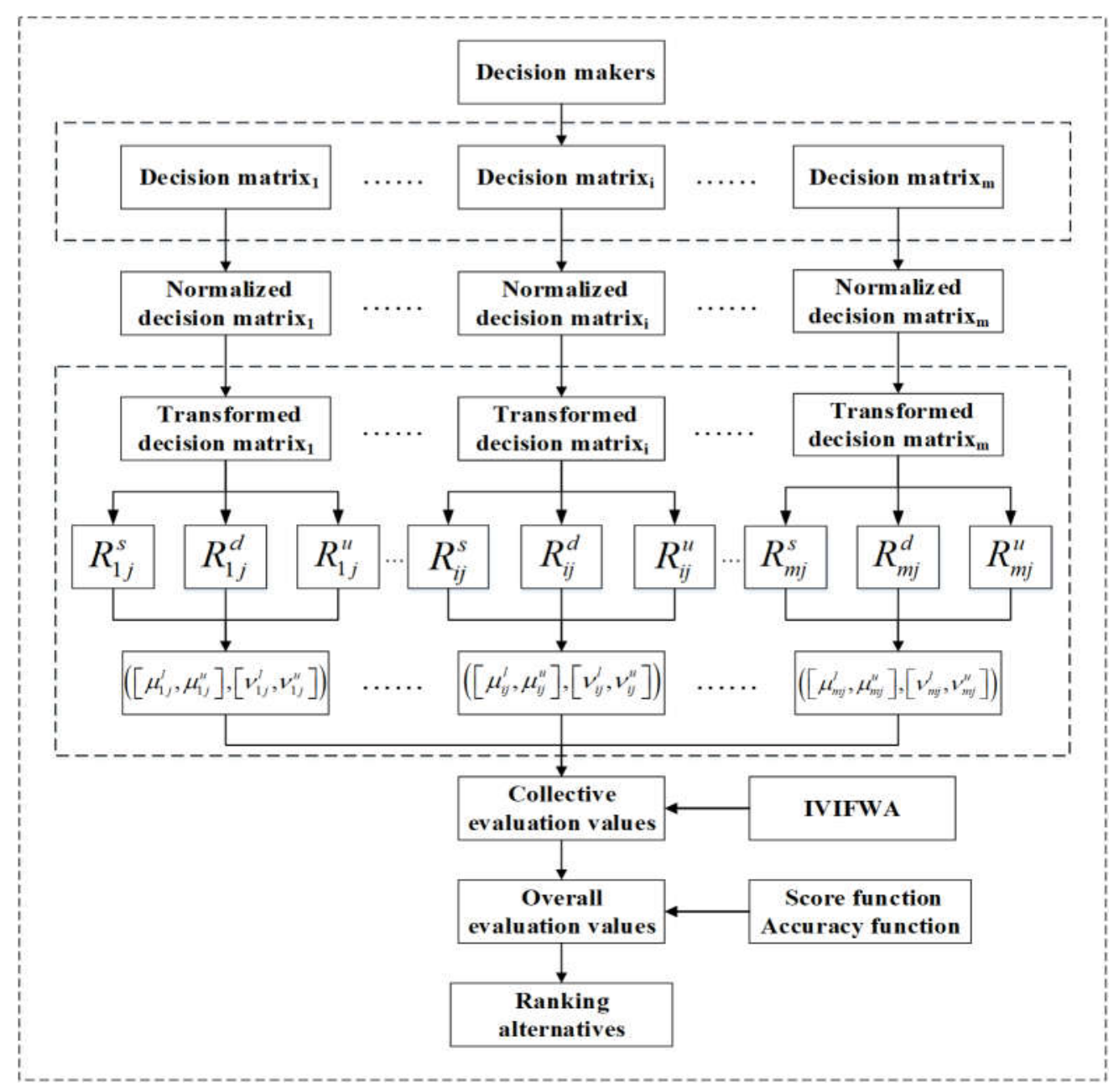

Figure 1 The framework of proposed MADM algorithm

4.2 Comparison with the counter examples

4.2.1 Comparison with the example of incorrect preference ranking

The same assumptions as those in the example of incorrect preference ranking, we apply the proposed method to deal with the first counter example, shown as follows:

Step 1: The decision matrix (DM) offered by decision-makers has to be described in Example 3.1, as follows:

Table 13 Decision matrix $X_{i}$

\begin{tabular}{cccc}
\hline Decision matrices & DMs & $u_{1}$ & $u_{2}$ \\
\hline$X_{1}$ & $d_{1}$ & {$[70,100]$} & {$[50,60]$} \\
& $d_{2}$ & {$[30,35]$} & {$[40,50]$} \\
& $d_{3}$ & {$[50,60]$} & {$[30,50]$} \\
\hline
\end{tabular}




\begin{tabular}{llll}
\hline & $d_{4}$ & {$[40,50]$} & {$[40,60]$} \\
$X_{2}$ & {$[40,50]$} & {$[50,60]$} \\
& $d_{1}$ & {$[30,35]$} & {$[45,55]$} \\
& $d_{2}$ & {$[40,50]$} & {$[40,60]$} \\
& $d_{3}$ & {$[60,90]$} & {$[50,60]$} \\
\hline
\end{tabular}

Step 2: According to Eq. (29), the attribute values in the decision matrix $X_{i}$ can be normalized to matrix $Y_{i}$, which are shown in Table 14.

Table 14 Normalized decision matrix $Y_{i}$

\begin{tabular}{cccc}
\hline Decision matrices & DMs & $u_{1}$ & $u_{2}$ \\
\hline$Y_{1}$ & $d_{1}$ & {$[0.70,1.00]$} & {$[0.83,1.00]$} \\
& $d_{2}$ & {$[0.30,0.35]$} & {$[0.67,0.83]$} \\
& $d_{3}$ & {$[0.50,0.60]$} & {$[0.50,0.83]$} \\
& $d_{4}$ & {$[0.40,0.50]$} & {$[0.67,1.00]$} \\
$Y_{2}$ & $d_{1}$ & {$[0.40,0.50]$} & {$[0.83,1.00]$} \\
& $d_{2}$ & {$[0.30,0.35]$} & {$[0.75,0.92]$} \\
& $d_{3}$ & {$[0.40,0.50]$} & {$[0.67,1.00]$} \\
& $d_{4}$ & {$[0.60,0.90]$} & {$[0.83,1.00]$} \\
\hline
\end{tabular}

Step 3: Based on Eq. (19) and the normalized matrix $Y_{i}$, We can construct the transformed matrix $R_{i}$ as Table 15.

Table 15 Transformed decision matrix $R_{i}$

\begin{tabular}{cccc}
\hline Decision matrices & DMs & $u_{1}$ & $u_{2}$ \\
\hline$R_{1}$ & $d_{1}$ & 0.850 & 0.9167 \\
& $d_{2}$ & 0.325 & 0.7500 \\
& $d_{3}$ & 0.550 & 0.6667 \\
& $d_{4}$ & 0.450 & 0.8333 \\
\hline
\end{tabular}




\begin{tabular}{llll}
\hline$R_{2}$ & $d_{1}$ & 0.450 & 0.9167 \\
& $d_{2}$ & 0.325 & 0.8333 \\
$d_{3}$ & 0.450 & 0.8333 \\
$d_{4}$ & 0.750 & 0.9167 \\
\hline
\end{tabular}

Step 4: By Eqs. (31)-(32) and the transformed decision matrix $R_{i}$, the satisfaction set, dissatisfaction set and the uncertainty set are collected as follows:

$$
\begin{aligned}
& R_{11}^{s}=\{0.850,0.550\}, R_{11}^{d}=\{0.325,0.450\}, R_{11}^{u}=\varnothing \\
& R_{12}^{s}=\{0.9167,0.7500,0.6667,0.8333\}, R_{12}^{d}=\varnothing, R_{12}^{u}=\varnothing \\
& R_{21}^{s}=\{0.750\}, R_{21}^{d}=\{0.450,0.325,0.450\}, R_{21}^{u}=\varnothing \\
& R_{22}^{s}=\{0.9167,0.8333,0.8333,0.9167\}, R_{22}^{d}=\varnothing, R_{22}^{u}=\varnothing
\end{aligned}
$$

Step 5-6: Using Eqs. (25)-(26), and Eqs. (34)-(35), the corresponding parameters of alternatives can be calculated, which are summarized in Table 16-17.

Table 16 The parameters of attributes of alternative $A_{1}$

\begin{tabular}{ccccccc}
\hline Attributes & Parameters & Values & Parameters & Values & Parameters & Values \\
\hline$u_{1}$ & $\xi_{11}^{l}$ & 0.5500 & $\zeta_{11}^{u}$ & 0.4500 & $\varsigma_{11}^{l}$ & 0.1000 \\
& $\xi_{11}^{u}$ & 0.8500 & $\kappa_{11}^{l}$ & 0.1000 & $\varsigma_{11}^{u}$ & 0.3500 \\
& $\zeta_{11}^{l}$ & 0.3250 & $\kappa_{11}^{u}$ & 0.7000 & $\tau_{11}$ & 1.2500 \\
$u_{2}$ & $\xi_{12}^{l}$ & 0.6667 & $\zeta_{12}^{u}$ & 0.5000 & $\varsigma_{2}^{1 l}$ & 0.0000 \\
& $\xi_{12}^{u}$ & 0.9167 & $\kappa_{12}^{l}$ & 0.3330 & $\varsigma_{2}^{1 u}$ & 0.0000 \\
& $\zeta_{12}^{l}$ & 0.5000 & $\kappa_{12}^{u}$ & 0.8334 & $\tau_{12}$ & 1.1664 \\
\hline
\end{tabular}

Table 17 The parameters of attributes of alternative $A_{2}$

\begin{tabular}{ccccccc}
\hline Attributes & Parameters & Values & Parameters & Values & Parameters & Values \\
\hline$u_{1}$ & $\xi_{21}^{l}$ & 0.7500 & $\zeta_{21}^{u}$ & 0.4500 & $\varsigma_{21}^{l}$ & 0.1000 \\
& $\xi_{21}^{u}$ & 0.7500 & $\kappa_{21}^{l}$ & 0.5000 & $\varsigma_{21}^{u}$ & 0.3500 \\
\hline
\end{tabular}




\begin{tabular}{lllllll}
\hline & $\zeta_{21}^{l}$ & 0.3250 & $\kappa_{21}^{u}$ & 0.5000 & $\tau_{21}$ & 1.4500 \\
$u_{2}$ & $\xi_{22}^{l}$ & 0.8333 & $\zeta_{22}^{u}$ & 0.5000 & $\varsigma_{2}^{2 l}$ & 0.0000 \\
$\xi_{22}^{u}$ & 0.9167 & $\kappa_{22}^{l}$ & 0.6666 & $\varsigma_{2}^{2 u}$ & 0.0000 \\
$\zeta_{22}^{l}$ & 0.5000 & $\kappa_{22}^{u}$ & 0.8334 & $\tau_{22}$ & 1.5000 \\
\hline
\end{tabular}

Step 7: As example 3.2.1, we let the weight of each attribute to be $w_{1}=w_{2}=0.5$. The collective evaluation of each alternative characterized by IVIFNs in Table 18 is aggregated by Eqs. (36) $-(37)$.

Table 18 The collective evaluation of each alternatives $A_{i}$

\begin{tabular}{ccc}
\hline Alternatives & $u_{1}$ & $u_{2}$ \\
\hline$A_{1}$ & $([0.0333,0.2333],[0.0333,0.1167])$ & $([0.2222,0.5556],[0,0])$ \\
$A_{2}$ & $([0.0833,0.0833],[0.0500,0.1750])$ & $([0.4444,0.5556],[0,0])$ \\
\hline
\end{tabular}

Step 8-9: The score of the overall evaluation and the preference order of each attribute are determined by Definition 2.16 and Definition 2.18, which are shown in Table 19.

Table 19 The score of the overall evaluation and the preference order of each alternatives $A_{i}$

\begin{tabular}{cccc}
\hline Alternatives & overall evaluation & Scores & Ranking \\
\hline$A_{1}$ & $([0.1329,0.4163],[0,0])$ & -0.1078 & 2 \\
$A_{2}$ & $([0.2863,0.3617],[0,0])$ & -0.0151 & 1 \\
\hline
\end{tabular}

4.2.2 Comparison with the example of illogical parameters and faulty evaluation results

The same assumptions as those in the example of illogical parameters and faulty evaluation results, we use the proposed MADM method to handle the second counter example, shown as follows:

Step 1: The decision matrix (DM) offered by decision-makers has to be described in Example 3.2, as follows:

Table 20 Decision matrix $X_{i}$

\begin{tabular}{llll} 
Decision matrices & DMs & $u_{1}$ & $u_{2}$ \\
\hline
\end{tabular}




\begin{tabular}{cccc}
\hline$X_{1}$ & $d_{1}$ & {$[70,80]$} & {$[8,10]$} \\
& $d_{2}$ & {$[80,85]$} & {$[9,10]$} \\
& $d_{3}$ & {$[90,100]$} & {$[8,9]$} \\
$d_{4}$ & {$[70,75]$} & {$[9,10]$} \\
$X_{2}$ & {$[65,80]$} & {$[8,9]$} \\
& $d_{1}$ & {$[75,85]$} & {$[1,2]$} \\
& $d_{2}$ & {$[90,95]$} & {$[1,2]$} \\
& $d_{3}$ & {$[65,75]$} & {$[1,3]$} \\
\hline
\end{tabular}

Step 2: According to Eq. (29), the attribute values in the decision matrix $X_{i}$ can be normalized to matrix $Y_{i}$, which are shown in Table 21.

Table 21 Normalized decision matrix $Y_{i}$

\begin{tabular}{cccc}
\hline Decision matrices & DMs & $u_{1}$ & $u_{2}$ \\
\hline$Y_{1}$ & $d_{1}$ & {$[0.70,0.80]$} & {$[0.08,0.10]$} \\
& $d_{2}$ & {$[0.80,0.85]$} & {$[0.09,0.10]$} \\
& $d_{3}$ & {$[0.90,1.00]$} & {$[0.08,0.09]$} \\
& $d_{4}$ & {$[0.70,0.75]$} & {$[0.09,0.10]$} \\
$Y_{2}$ & $d_{1}$ & {$[0.65,0.80]$} & {$[0.08,0.09]$} \\
& $d_{2}$ & {$[0.75,0.85]$} & {$[0.01,0.02]$} \\
& $d_{3}$ & {$[0.9,0.95]$} & {$[0.01,0.02]$} \\
& $d_{4}$ & {$[0.65,0.75]$} & {$[0.01,0.03]$} \\
\hline
\end{tabular}

Step 3: Based on Eq. (19) and the normalized matrix $Y_{i}$, We can construct the transformed matrix $R_{i}$ as Table 22 .

Table 22 Transformed decision matrix $R_{i}$

\begin{tabular}{cccc}
\hline Decision matrices & DMs & $u_{1}$ & $u_{2}$ \\
\hline$R_{1}$ & $d_{1}$ & 0.750 & 0.090 \\
& $d_{2}$ & 0.825 & 0.095 \\
\hline
\end{tabular}




\begin{tabular}{llll}
\hline & $d_{3}$ & 0.950 & 0.085 \\
$d_{4}$ & 0.725 & 0.095 \\
$R_{2}$ & 0.725 & 0.085 \\
& $d_{1}$ & 0.800 & 0.015 \\
& $d_{2}$ & 0.925 & 0.015 \\
& $d_{3}$ & 0.700 & 0.020 \\
\hline
\end{tabular}

Step 4: By Eqs. (31)-(32) and the transformed decision matrix $R_{i}$, the satisfaction set, dissatisfaction set and the uncertainty set are collected as follows:

$$
\begin{aligned}
& R_{11}^{s}=\{0.750,0.825,0.950,0.725\}, R_{11}^{d}=\varnothing, R_{11}^{u}=\varnothing \\
& R_{12}^{s}=\varnothing, R_{12}^{d}=\{0.085,0.015,0.015,0.020\}, R_{12}^{u}=\varnothing \\
& R_{21}^{s}=\{0.725,0.800,0.925,0.700\}, R_{21}^{d}=\varnothing, R_{21}^{u}=\varnothing \\
& R_{22}^{s}=\varnothing, R_{22}^{d}=\{0.090,0.095,0.085,0.095\}, R_{22}^{u}=\varnothing
\end{aligned}
$$

Step 5-6: Using Eqs. (25)-(26), and Eqs. (34)-(35), the corresponding parameters of alternatives can be calculated, which are summarized in Table 23-24.

\begin{tabular}{|c|c|c|c|c|c|c|}
\hline Attributes & Parameters & Values & Parameters & Values & Parameters & Values \\
\hline
\end{tabular}

Table 23 The parameters of attributes of alternative $A_{1}$

\begin{tabular}{ccccccc}
\hline Attributes & Parameters & Values & Parameters & Values & Parameters & Values \\
\hline$u_{1}$ & $\xi_{11}^{l}$ & 0.725 & $\zeta_{11}^{u}$ & 0.500 & $\varsigma_{11}^{l}$ & 0.000 \\
& $\xi_{11}^{u}$ & 0.950 & $\kappa_{11}^{l}$ & 0.450 & $\varsigma_{11}^{u}$ & 0.000 \\
& $\zeta_{11}^{l}$ & 0.500 & $\kappa_{11}^{u}$ & 0.900 & $\tau_{11}$ & 1.350 \\
$u_{2}$ & $\xi_{12}^{l}$ & 0.500 & $\zeta_{12}^{u}$ & 0.095 & $\varsigma_{2}^{l l}$ & 0.810 \\
& $\xi_{12}^{u}$ & 0.500 & $\kappa_{12}^{l}$ & 0.000 & $\varsigma_{2}^{1 u}$ & 0.830 \\
& $\zeta_{12}^{l}$ & 0.085 & $\kappa_{12}^{u}$ & 0.000 & $\tau_{12}$ & 1.640 \\
\hline
\end{tabular}

Table 24 The parameters of attributes of alternative $A_{2}$ 


\begin{tabular}{lllllll}
\hline$u_{1}$ & $\xi_{21}^{l}$ & 0.700 & $\zeta_{21}^{u}$ & 0.500 & $\varsigma_{21}^{l}$ & 0.000 \\
& $\xi_{21}^{u}$ & 0.925 & $\kappa_{21}^{l}$ & 0.400 & $\varsigma_{21}^{u}$ & 0.000 \\
& $\zeta_{21}^{l}$ & 0.500 & $\kappa_{21}^{u}$ & 0.850 & $\tau_{21}$ & 1.250 \\
& $\xi_{22}^{l}$ & 0.500 & $\zeta_{22}^{u}$ & 0.085 & $\varsigma_{2}^{2 l}$ & 0.830 \\
& $\xi_{22}^{u}$ & 0.500 & $\kappa_{22}^{l}$ & 0.000 & $\varsigma_{2}^{2 u}$ & 0.970 \\
& $\zeta_{22}^{l}$ & 0.015 & $\kappa_{22}^{u}$ & 0 & $\tau_{22}$ & 1.8 \\
\hline
\end{tabular}

Step 7: As example 3.2.2, we let the weight of each attribute to be $w_{1}=w_{2}=0.5$. The collective evaluation of each alternative characterized by IVIFNs in Table 25 is aggregated by Eqs. (36) $-(37)$.

Table 25 The collective evaluation of each alternatives $A_{i}$

\begin{tabular}{ccc}
\hline Alternatives & $u_{1}$ & $u_{2}$ \\
\hline$A_{1}$ & $([0.2500,0.5000],[0,0])$ & $([0,0],[0.4500,0.4611])$ \\
$A_{2}$ & $([0.2222,0.4722],[0,0])$ & $([0,0],[0.4611,0.5389])$ \\
\hline
\end{tabular}

Step 8-9: The score of the overall evaluation and the preference order of each attribute are determined by Definition 2.16 and Definition 2.18, which are shown in Table 26.

Table 26 The score of the overall evaluation and the preference order of each alternatives $A_{i}$

\begin{tabular}{cccc}
\hline Alternatives & overall evaluation & Scores & Ranking \\
\hline$A_{1}$ & $([0.1340,0.2929],[0,0])$ & -0.1875 & 1 \\
$A_{2}$ & $([0.1181,0.2735],[0,0])$ & -0.2143 & 2 \\
\hline
\end{tabular}

It is apparent to find that the preference order of alternatives is $A_{1} \succ A_{2}$ as before. Therefore, the improved MADM method we proposed can conquer the shortcoming of Yue's MADM method [27] for ranking of alternatives effectively.

\subsection{Illustrative example}

We conducted a comprehensive evaluation of the air quality of Fuzhou, Nanping and Zhangzhou in Fujian province in 2019. Let Fuzhou, Nanping and Zhangzhou are considered 
$A=\{$ Fuzhou, Nanping and Zhangzhou $\}$ to be the set of the alternatives and $U=\left\{u_{1}, u_{2}, u_{3}\right\}=\left\{S O_{2}, N O_{2}, P M_{10}\right\}$ be the set of attributes. Let the air quality of each quarter as DM, $D=\{$ First quarter, Second quarter, Third quarter,Fourth quarter $\}$.

The weight vector $w=\left(\frac{1}{3}, \frac{1}{3}, \frac{1}{3}\right)^{T}$ of $\left\{S O_{2}, N O_{2}, P M_{10}\right\}$, which is determined according to the Implementing Details for Urban Environmental Comprehensive Treatment and Quantitative Examination during the 12th Five-Year Plan (Ministry of Ecology and Environment of the People's Republic of China, No. 1302) [30]. The air quality data [31] is shown in

Table 27

Since the lower the values of the three indicators, the better the air quality and the range from 0 to 300 is the condition for healthy people to be able to exercise outdoors. According to Step 2, we first normalize data in

-29 to the corresponding

-32 by Eq. (30). Then we further

Table 27

Table 30

transform the normalized matrix into the transformed decision matrix by Step 3, shown in

Table

-35. Based on Eqs. (31)-(33) in Step 4, we can get the satisfaction, dissatisfaction and 33

uncertainty set of the corresponding properties for each alternative.

According to Eqs. (25-26) and Eqs. (34)-(37) in Steps 5-7, the parameters and collective evaluation of each attribute are calculated in Table 36-39. The overall evaluation results, which are characterized by IVIFNs of air quality, are calculated by Step 8, and the scores and orders of each alternative are summarized in Table 40. Their sorting is $A_{2} \succ A_{1} \succ A_{3}$. This result is consistent with the official air quality ranking, which proves the feasibility of the proposed algorithm. The interval-values of attributes for each alternative and each alternative's scores are illustrated in Figure 2 and Figure 3, respectively.

Many practical problems are often characterized by MADM. In this section, taking thousands of air quality data generated every day as an example, it is very difficult or costly to store and analyze large-scale data directly. However, it becomes easier to save and process air information for a period of time through interval values. In this paper, we have developed a straightforward and practical aggregation technique to convert interval values into an IVIFN and apply the proposed MADM method to air quality assessment.

Table 27 The air quality in Fuzhou City of 2019

\begin{tabular}{ccccc}
\hline Decision matrices & $\mathrm{DMs}$ & $\mathrm{SO}_{2}$ & $\mathrm{NO}$ & $P M_{10}$ \\
\hline$X_{1}$ & $d_{1}$ & {$[4,5]$} & {$[21,33]$} & {$[32,46]$} \\
\hline
\end{tabular}




\begin{tabular}{lllll}
\hline$d_{2}$ & {$[4,5]$} & {$[19,27]$} & {$[35,53]$} \\
$d_{3}$ & {$[4,4]$} & {$[13,17]$} & {$[32,37]$} \\
$d_{4}$ & {$[5,6]$} & {$[16,33]$} & {$[46,49]$} \\
\hline
\end{tabular}

Table 28 The air quality in Nanping City of 2019

\begin{tabular}{ccccc}
\hline Decision matrices & $\mathrm{DMs}$ & $\mathrm{SO}_{2}$ & $\mathrm{NO}_{2}$ & $P M_{10}$ \\
\hline$X_{2}$ & $d_{1}$ & {$[6,8]$} & {$[12,23]$} & {$[20,34]$} \\
& $d_{2}$ & {$[6,7]$} & {$[12,15]$} & {$[23,34]$} \\
& $d_{3}$ & {$[6,8]$} & {$[9,14]$} & {$[25,32]$} \\
& $d_{4}$ & {$[8,9]$} & {$[18,27]$} & {$[38,43]$} \\
\hline
\end{tabular}

Table 29 The air quality in Zhangzhou City of 2019

\begin{tabular}{ccccc}
\hline Decision matrices & $\mathrm{DMs}$ & $\mathrm{SO}_{2}$ & $\mathrm{NO}_{2}$ & $P M_{10}$ \\
\hline$X_{3}$ & $d_{1}$ & {$[4,8]$} & {$[24,39]$} & {$[57,76]$} \\
& $d_{2}$ & {$[8,10]$} & {$[18,29]$} & {$[26,55]$} \\
& $d_{3}$ & {$[6,8]$} & {$[16,22]$} & {$[35,50]$} \\
& $d_{4}$ & {$[4,8]$} & {$[24,39]$} & {$[57,76]$} \\
\hline
\end{tabular}

Table 30 Normalized air quality in Fuzhou City of 2019

\begin{tabular}{ccccc}
\hline Decision matrices & $\mathrm{DMs}$ & $\mathrm{SO}_{2}$ & $\mathrm{NO}_{2}$ & $P M_{10}$ \\
\hline$Y_{1}$ & $d_{1}$ & {$[0.9833,0.9867]$} & {$[0.8900,0.9300]$} & {$[0.8467,0.8933]$} \\
& $d_{2}$ & {$[0.9833,0.9867]$} & {$[0.9100,0.9367]$} & {$[0.8233,0.8833]$} \\
& $d_{3}$ & {$[0.9867,0.9867]$} & {$[0.9433,0.9567]$} & {$[0.8767,0.8933]$} \\
& $d_{4}$ & {$[0.9800,0.9833]$} & {$[0.8900,0.9467]$} & {$[0.8367,0.8467]$}
\end{tabular}

Table 31 Normalized air quality in Nanping City of 2019

\begin{tabular}{ccccc}
\hline Decision matrices & $\mathrm{DMs}$ & $\mathrm{SO}_{2}$ & $\mathrm{NO}_{2}$ & $P M_{10}$ \\
\hline$Y_{2}$ & $d_{1}$ & {$[0.9733,0.9800]$} & {$[0.9233,0.9600]$} & {$[0.8867,0.9333]$} \\
& $d_{2}$ & {$[0.9767,0.9800]$} & {$[0.9500,0.9600]$} & {$[0.8867,0.9233]$} \\
\hline
\end{tabular}




$\begin{array}{llll}d_{3} & {[0.9733,0.9800]} & {[0.9533,0.9700]} & {[0.8933,0.9167]} \\ d_{4} & {[0.9700,0.9733]} & {[0.9100,0.9400]} & {[0.8567,0.8733]}\end{array}$

Table 32 Normalized air quality in Zhangzhou City of 2019

\begin{tabular}{ccccc}
\hline Decision matrices & $\mathrm{DMs}$ & $\mathrm{SO}_{2}$ & $\mathrm{NO}_{2}$ & $P M_{10}$ \\
\hline$Y_{3}$ & $d_{1}$ & {$[0.9733,0.9867]$} & {$[0.8700,0.9200]$} & {$[0.7467,0.8100]$} \\
& $d_{2}$ & {$[0.9667,0.9733]$} & {$[0.9033,0.9400]$} & {$[0.8167,0.9133]$} \\
& $d_{3}$ & {$[0.9733,0.9800]$} & {$[0.9267,0.9467]$} & {$[0.8333,0.8833]$} \\
& $d_{4}$ & {$[0.9633,0.9700]$} & {$[0.8633,0.9133]$} & {$[0.7700,0.7800]$} \\
\hline
\end{tabular}

Table 33 Transformed air quality in Fuzhou City of 2019

\begin{tabular}{ccccc}
\hline Decision matrices & $\mathrm{DMs}$ & $\mathrm{SO}_{2}$ & $\mathrm{NO}_{2}$ & $P M_{10}$ \\
\hline$R_{1}$ & $d_{1}$ & 0.9850 & 0.9100 & 0.8700 \\
& $d_{2}$ & 0.9850 & 0.9234 & 0.8533 \\
& $d_{3}$ & 0.9867 & 0.9500 & 0.8850 \\
& $d_{4}$ & 0.9816 & 0.9184 & 0.8417 \\
\hline
\end{tabular}

Table 34 Transformed air quality in Nanping City of 2019

\begin{tabular}{ccccc}
\hline Decision matrices & $\mathrm{DMs}$ & $\mathrm{SO}_{2}$ & $\mathrm{NO}_{2}$ & $P M_{10}$ \\
\hline$R_{2}$ & $d_{1}$ & 0.9767 & 0.9416 & 0.9100 \\
& $d_{2}$ & 0.9784 & 0.9550 & 0.9050 \\
& $d_{3}$ & 0.9767 & 0.9617 & 0.9050 \\
& $d_{4}$ & 0.9717 & 0.9250 & 0.8650 \\
\hline
\end{tabular}

Table 35 Transformed air quality in Zhangzhou City of 2019

\begin{tabular}{ccccc}
\hline Decision matrices & $\mathrm{DMs}$ & $\mathrm{SO}_{2}$ & $\mathrm{NO}_{2}$ & $P M_{10}$ \\
\hline$R_{3}$ & $d_{1}$ & 0.9800 & 0.8950 & 0.7784 \\
& $d_{2}$ & 0.9700 & 0.9216 & 0.8650 \\
\hline
\end{tabular}




\begin{tabular}{llll}
\hline$d_{3}$ & 0.9767 & 0.9367 & 0.8583 \\
$d_{4}$ & 0.9667 & 0.8883 & 0.7750 \\
\hline
\end{tabular}

$$
\begin{aligned}
& R_{11}^{s}=\{0.9850,0.9850,0.9867,0.9816\}, R_{11}^{d}=\varnothing, R_{11}^{u}=\varnothing \\
& R_{12}^{s}=\{0.9100,0.9234,0.9500,0.9184\}, R_{12}^{d}=\varnothing, R_{12}^{u}=\varnothing \\
& R_{13}^{s}=\{0.8700,0.8533,0.8850,0.8417\}, R_{12}^{d}=\varnothing, R_{12}^{u}=\varnothing \\
& R_{21}^{s}=\{0.9767,0.9784,0.9767,0.9717\}, R_{21}^{d}=\varnothing, R_{21}^{u}=\varnothing \\
& R_{22}^{s}=\{0.9416,0.9550,0.9617,0.9250\}, R_{22}^{d}=\varnothing, R_{22}^{u}=\varnothing \\
& R_{23}^{s}=\{0.9100,0.9050,0.9050,0.8650\}, R_{22}^{d}=\varnothing, R_{22}^{u}=\varnothing \\
& R_{31}^{s}=\{0.9800,0.9700,0.9767,0.9667\}, R_{31}^{d}=\varnothing, R_{31}^{u}=\varnothing \\
& R_{32}^{s}=\{0.8950,0.9216,0.9367,0.8883\}, R_{32}^{d}=\varnothing, R_{32}^{u}=\varnothing \\
& R_{33}^{s}=\{0.7784,0.8650,0.8583,0.7750\}, R_{32}^{d}=\varnothing, R_{32}^{u}=\varnothing
\end{aligned}
$$

Table 36 The parameters of attributes of alternative $A_{1}$

\begin{tabular}{ccccccc}
\hline Attributes & Parameters & Values & Parameters & Values & Parameters & Values \\
\hline$u_{1}$ & $\xi_{11}^{l}$ & 0.9816 & $\zeta_{11}^{u}$ & 0.5000 & $\varsigma_{11}^{l}$ & 0.0000 \\
& $\xi_{11}^{u}$ & 0.9867 & $\kappa_{11}^{l}$ & 0.9632 & $\varsigma_{11}^{u}$ & 0.0000 \\
& $\zeta_{11}^{l}$ & 0.5000 & $\kappa_{11}^{u}$ & 0.9734 & $\tau_{11}$ & 1.9366 \\
$u_{2}$ & $\xi_{12}^{l}$ & 0.9100 & $\zeta_{12}^{u}$ & 0.5000 & $\varsigma_{2}^{1 l}$ & 0.0000 \\
& $\xi_{12}^{u}$ & 0.9500 & $\kappa_{12}^{l}$ & 0.8200 & $\varsigma_{2}^{1 u}$ & 0.0000 \\
& $\zeta_{12}^{l}$ & 0.5000 & $\kappa_{12}^{u}$ & 0.9000 & $\tau_{12}$ & 1.7200 \\
$u_{3}$ & $\xi_{13}^{l}$ & 0.8417 & $\zeta_{13}^{u}$ & 0.5000 & $\varsigma_{3}^{1 l}$ & 0.0000 \\
& $\xi_{13}^{u}$ & 0.8850 & $\kappa_{13}^{l}$ & 0.6834 & $\varsigma_{3}^{1 u}$ & 0.0000 \\
& $\zeta_{13}^{l}$ & 0.5000 & $\kappa_{13}^{u}$ & 0.7700 & $\tau_{13}$ & 1.4534 \\
\hline
\end{tabular}

Table 37 The parameters of attributes of alternative $A_{2}$

\begin{tabular}{ccccccc}
\hline Attributes & Parameters & Values & Parameters & Values & Parameters & Values \\
\hline$u_{1}$ & $\xi_{21}^{l}$ & 0.9717 & $\zeta_{21}^{u}$ & 0.5000 & $\varsigma_{21}^{l}$ & 0.0000
\end{tabular}




\begin{tabular}{|c|c|c|c|c|c|c|}
\hline & $\xi_{21}^{u}$ & 0.9784 & $\kappa_{21}^{l}$ & 0.9434 & $\varsigma_{21}^{u}$ & 0.0000 \\
\hline & $\zeta_{21}^{l}$ & 0.5000 & $\kappa_{21}^{u}$ & 0.9568 & $\tau_{21}$ & 1.9002 \\
\hline$u_{2}$ & $\xi_{22}^{l}$ & 0.9250 & $\zeta_{22}^{u}$ & 0.5000 & $\varsigma_{2}^{2 l}$ & 0.0000 \\
\hline & $\xi_{22}^{u}$ & 0.9617 & $\kappa_{22}^{l}$ & 0.8500 & $\varsigma_{2}^{2 u}$ & 0.0000 \\
\hline & $\zeta_{22}^{l}$ & 0.5000 & $\kappa_{22}^{u}$ & 0.9234 & $\tau_{22}$ & 1.4234 \\
\hline$u_{3}$ & $\xi_{23}^{l}$ & 0.8650 & $\zeta_{23}^{u}$ & 0.5000 & $\varsigma_{3}^{2 l}$ & 0.0000 \\
\hline & $\xi_{23}^{u}$ & 0.9100 & $\kappa_{23}^{l}$ & 0.7300 & $\varsigma_{3}^{2 u}$ & 0.0000 \\
\hline & $\zeta_{23}^{l}$ & 0.5000 & $\kappa_{23}^{u}$ & 0.8200 & $\tau_{23}$ & 1.5500 \\
\hline
\end{tabular}

Table 38 The parameters of attributes of alternative $A_{3}$

\begin{tabular}{ccccccc}
\hline Attributes & Parameters & Values & Parameters & Values & Parameters & Values \\
\hline$u_{1}$ & $\xi_{31}^{l}$ & 0.9667 & $\zeta_{31}^{u}$ & 0.5000 & $\varsigma_{31}^{l}$ & 0.0000 \\
& $\xi_{31}^{u}$ & 0.9800 & $\kappa_{31}^{l}$ & 0.9334 & $\varsigma_{31}^{u}$ & 0.0000 \\
& $\zeta_{31}^{l}$ & 0.5000 & $\kappa_{31}^{u}$ & 0.9600 & $\tau_{31}$ & 1.8934 \\
$u_{2}$ & $\xi_{32}^{l}$ & 0.8883 & $\zeta_{32}^{u}$ & 0.5000 & $\varsigma_{2}^{3 l}$ & 0.0000 \\
& $\xi_{32}^{u}$ & 0.9367 & $\kappa_{32}^{l}$ & 0.7766 & $\varsigma_{2}^{3 u}$ & 0.0000 \\
& $\zeta_{32}^{l}$ & 0.5000 & $\kappa_{32}^{u}$ & 0.8734 & $\tau_{32}$ & 1.6500 \\
& $\xi_{33}^{l}$ & 0.7750 & $\zeta_{33}^{u}$ & 0.5000 & $\varsigma_{3}^{3 l}$ & 0.0000 \\
& $\xi_{33}^{u}$ & 0.8650 & $\kappa_{33}^{l}$ & 0.5500 & $\varsigma_{3}^{3 u}$ & 0.0000 \\
& $\zeta_{33}^{l}$ & 0.5000 & $\kappa_{33}^{u}$ & 0.7300 & $\tau_{33}$ & 1.2800 \\
\hline
\end{tabular}

Table 39 The collective evaluation of each alternative $A_{i}$

\begin{tabular}{cccc}
\hline Alternatives & $\mathrm{SO}_{2}$ & $\mathrm{NO}_{2}$ & $P M_{10}$ \\
\hline$A_{1}$ & $([0.4974,0.5025],[0,0])$ & $([0.4234,0.4647],[0,0])$ & $([0.3529,0.3976],[0,0])$ \\
$A_{2}$ & $([0.4871,0.4941],[0,0])$ & $([0.4389,0.4768],[0,0])$ & $([0.3769,0.4234],[0,0])$ \\
$A_{3}$ & $([0.4820,0.4957],[0,0])$ & $([0.4010,0.4510],[0,0])$ & $([0.2840,0.3769],[0,0])$ \\
\hline
\end{tabular}




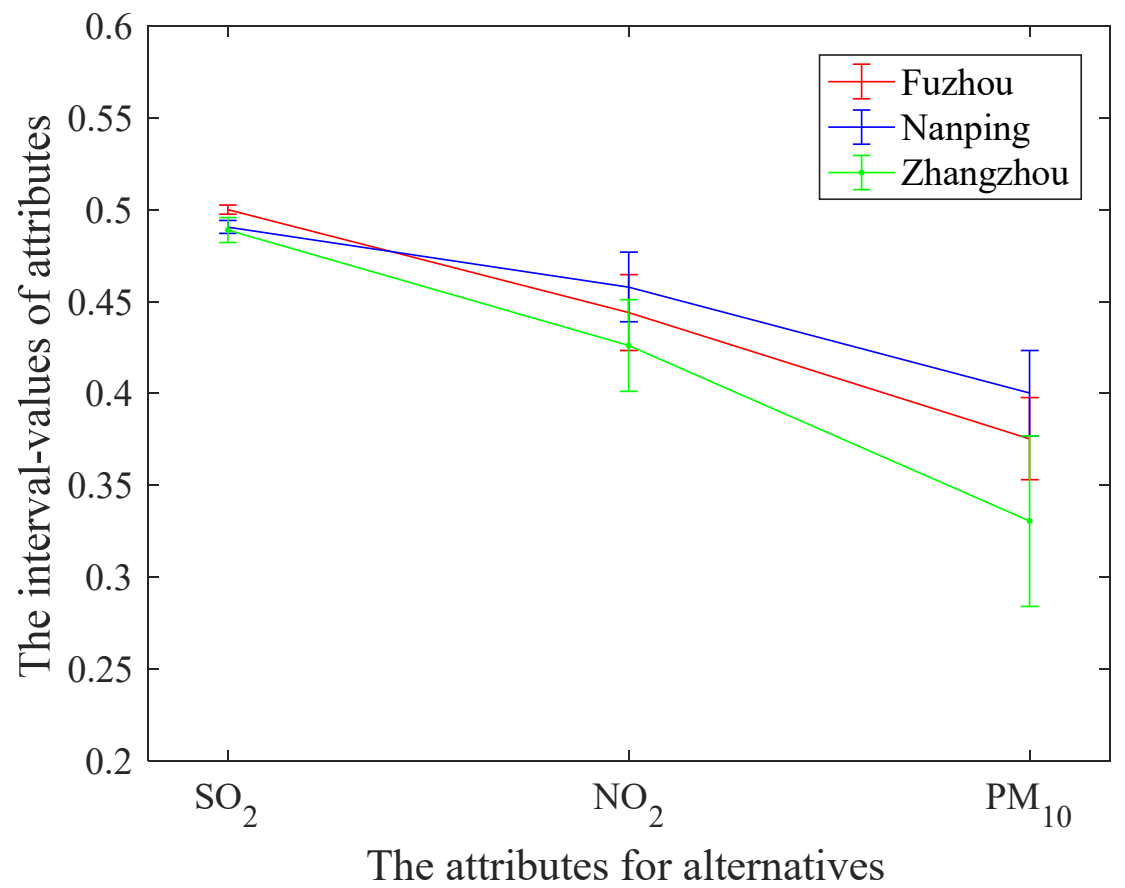

Figure 2 The interval-values of attributes for each alternative

Table 40 The score of the overall evaluation and the preference order of each alternatives $A_{i}$

\begin{tabular}{cccc}
\hline Alternatives & overall evaluation & Scores & Ranking \\
\hline$A_{1}$ & $([0.4276,0.4566],[0,0])$ & 0.1630 & 2 \\
$A_{2}$ & $([0.4361,0.4656],[0,0])$ & 0.1762 & 1 \\
$A_{3}$ & $([0.3943,0.4433],[0,0])$ & 0.1278 & 3 \\
\hline
\end{tabular}




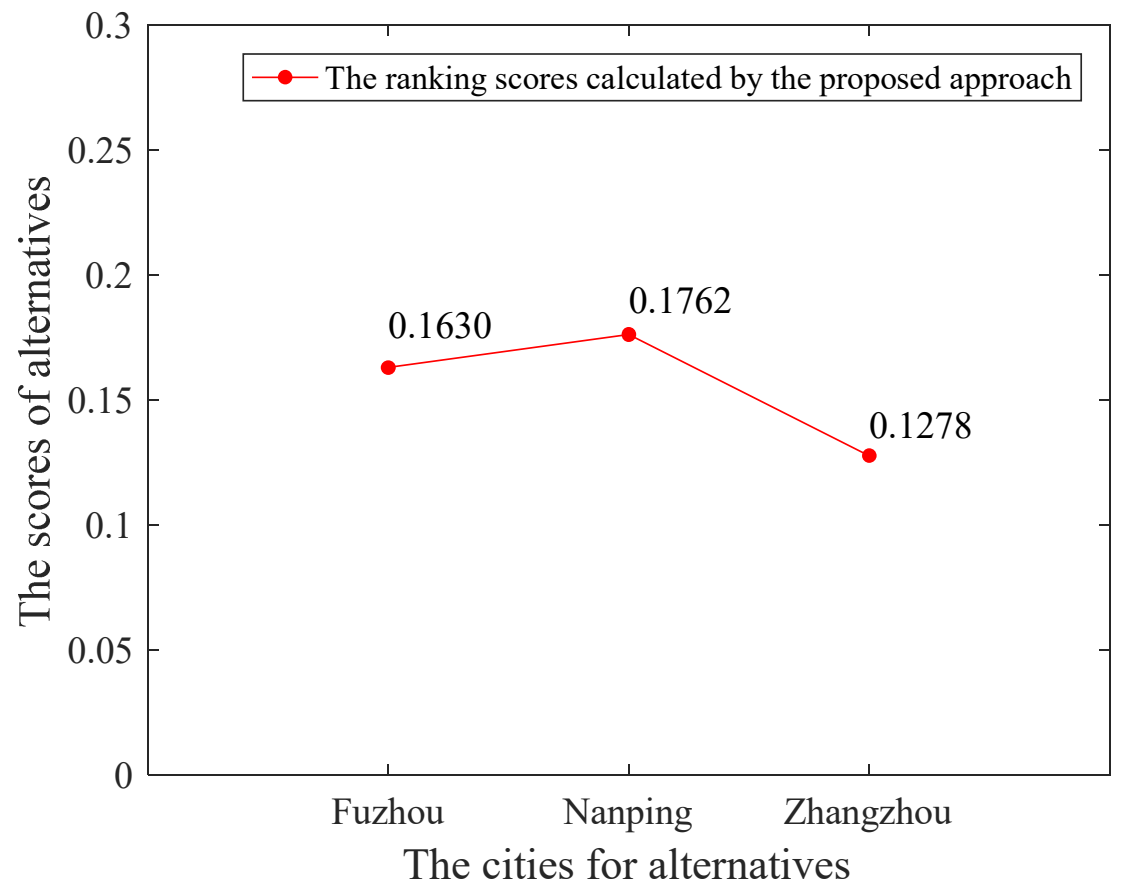

Figure 3 The scores of each alternative

\section{Conclusions}

In this paper, a novel MADM method has been developed, in which the attribute values are characterized by interval numbers. Moreover, attribute values of each alternative have been aggregated into an induced IVIFN for ranking under interval-valued intuitionistic fuzzy environment.

It is worth pointing out that: (1) the method has no restrictions on the data distribution, the sample size, and the number of attributes; (2) the ranking of the alternatives is straightforward and effective when the overall evaluation is quantified by the aggregated IVIFNs; (3) The richer the data, the more the superiority. Furthermore, the transformation is simple and can be performed easily on a computer. The major contributions of this paper are fourfold:

(1) The novel score function and accuracy function for ranking IVIFNs are proposed.

(2) Pointing out the problems of Yue's MADM method through counter-examples.

(3) A new methodology for MADM problems under interval-valued intuitionistic fuzzy situation is established.

(4) An instance analysis is provided to elucidate the reasonableness and efficiency of the proposed method.

The proposed method can be programmed and implemented easily on a computer. In the future, we will study the application of the proposed MADM method to different decision-making problems, such as big data analysis, pattern recognition, expert systems. 


\section{Funding}

This work was supported by the Natural Science Foundation of Fujian Province (No. 2020J01576), the Science and Technology Innovation Special Fund Project of Fujian agriculture and Forestry University (No. CXZX2017492) and the Construction Fund for Digital Fujian Big Data for Agriculture and Forestry (No. KJG18019A).

\section{Availability of data and material}

The data used to support the findings of this study are included within the article.

\section{Conflicts of interest/Competing interests}

We declare that we have no financial and personal relationships with other people or organizations that can inappropriately influence our work, there is no professional or other personal interest of any nature or kind in any product, service and/or company that could be construed as influencing the position presented in, or the review of, the manuscript entitled.

\section{Authors' contributions}

Jian Lin: Conceptualization, Methodology, Investigation, Writing - Original Draft, Software, Data Curation, Writing - Review \& Editing. Riqing Chen: Validation, Resources, Formal analysis, Writing - Review \& Editing. Jian Lin: Methodology, Writing - Original Draft, Resources, Writing - Review \& Editing.

\section{References}

[1] L. A. Zadeh. "Fuzzy Sets." Information \& Control 8 (1965): 338-353.

[2] J. Vanicek, I. Vrana,S. Aly. "Fuzzy Aggregation and Averaging for Group Decision Making: A Generalization and Survey." Knowledge-Based Systems 22 (2009): 79-84.

[3] G. Deschrijver,E. E. Kerre. "On the Composition of Intuitionistic Fuzzy Relations." Fuzzy Sets \& Systems 136 (2003): 333-361.

[4] O. López-Ortega,M. A. Rosales. "An Agent-Oriented Decision Support System Combining Fuzzy Clustering and the Ahp." Expert Systems with Applications 38 (2011): 8275-8284.

[5] K. T. Atanassov. "Intuitionistic Fuzzy Sets." Fuzzy Sets \& Systems 20 (1986): 87-96.

[6] K. T. Atanassov. "Intuitionistic Fuzzy Sets." Intuitionistic Fuzzy Sets. Springer, 1999. 1-137.

[7] K. Atanassov. "Intuitionistic Fuzzy Sets." International Journal Bioautomation 20 (2016): 1.

[8] K. Atanassov,G. Gargov. "Interval Valued Intuitionistic Fuzzy Sets." Fuzzy Sets and Systems 31 (1989): 343-349.

[9] Z. Xu. "Methods for Aggregating Interval-Valued Intuitionistic Fuzzy Information and Their Application to Decision Making." Control \& Decision 22 (2007): 215-219.

[10] Z. Wang, K. W. Li,W. Wang. "An Approach to Multiattribute Decision Making with Interval-Valued Intuitionistic Fuzzy Assessments and Incomplete Weights." Information Sciences 179 (2009): 3026-3040. 
[11] J. Ye. "Multicriteria Fuzzy Decision-Making Method Based on a Novel Accuracy Function under Interval-Valued Intuitionistic Fuzzy Environment." Expert Systems with Applications 36 (2009): 6899-6902.

[12] V. L. G. Nayagam, S. Muralikrishnan,G. Sivaraman. "Multi-Criteria Decision-Making Method Based on Interval-Valued Intuitionistic Fuzzy Sets." Expert Systems With Applications 38 (2010): 1464-1467.

[13] D. YAO,J. MAO. "Decision-Making Methods for Interval-Valued Intuitionistic Fuzzy Information Based on Hesitancy Degree and Their Application." Journal of Hefei University ( Natural Sciences) 21 (2011): 1-4.

[14] Z. Wang,L. Niu,S. Mathamp, et al. "A New Scoring Function of Interval-Valued Intuitionistic Fuzzy Number and Its Application in Multi-Attribute Decision Making." Fuzzy Systems and $M$ athematics 27 (2013): 167-172.

[15] J. GAO, H. Liu,Y. Gu. "Interval-Valued Intuitionistic Fuzzy Multi-Criteria Decision-Making Method Based on Prospect Theory." Systems Engineering - Theory \& Practice 34 (2014): 3175-3181.

[16] M. Gao, T. Sun,J. Zhu. "Interval-Valued Intuitionistic Fuzzy Multiple Attribute Decision-Making Method Based on Revised Fuzzy Entropy and New Scoring Function." Control and Decision 31 (2016): 1757-1764.

[17] C. Y. Wang,S. M. Chen. "An Improved Multiattribute Decision Making Method Based on New Score Function of Interval-Valued Intuitionistic Fuzzy Values and Linear Programming Methodology." Information Sciences 411 (2017): 176-184.

[18] R. Gong,L. Ma. "A New Score Function and Accuracy Function of Interval-Valued Intuitionistic Fuzzy Number and Its Application." Systems Engineering-Theory \& Practice 39 (2019): 463-475.

[19] K. Kumar,S.-M. Chen. "Multiattribute Decision Making Based on Interval-Valued Intuitionistic Fuzzy Values, Score Function of Connection Numbers, and the Set Pair Analysis Theory." Information Sciences 551 (2021): 100-112.

[20] S.-M. Chen,W.-T. Liao. "Multiple Attribute Decision Making Using Beta Distribution of Intervals, Expected Values of Intervals, and New Score Function of Interval-Valued Intuitionistic Fuzzy Values." Information Sciences (2021).

[21] Z. Yue, Y. Jia,G. Ye. "An Approach for Multiple Attribute Group Decision Making Based on Intuitionistic Fuzzy Information." International Journal of Uncertainty, Fuzziness and Knowledge-Based Systems 17 (2009): 317-332.

[22] Z. Yue. "An Approach to Aggregating Interval Numbers into Interval-Valued Intuitionistic Fuzzy Information for Group Decision Making." Expert Systems with Applications 38 (2011): 6333-6338.

[23] Z. Yue,Y. Jia. "A Method to Aggregate Crisp Values into Interval-Valued Intuitionistic Fuzzy Information for Group Decision Making." Applied Soft Computing Journal 13 (2013): 2304-2317.

[24] J. Lin,Q. Zhang. "Note on Aggregating Crisp Values into Intuitionistic Fuzzy Number." Applied Mathematical Modelling 40 (2016): 10800-10808.

[25] S. P. Wan, J. Xu,J. Y. Dong. "Aggregating Decision Information into Interval-Valued Intuitionistic Fuzzy Numbers for Heterogeneous Multi-Attribute Group Decision Making." Knowledge Based Systems 113 (2016): 155-170.

[26] J. Xu, S. P. Wan,J. Y. Dong. "Aggregating Decision Information into Atanassov's Intuitionistic Fuzzy Numbers for Heterogeneous Multi-Attribute Group Decision Making." Applied Soft Computing 41 (2016): 331-351. 
[27] Yue,Zhongliang. "A Group Decision Making Approach Based on Aggregating Interval Data into Interval-Valued Intuitionistic Fuzzy Information." Applied Mathematical Modelling 38 (2014): 683-698.

[28] S. Liu,W. Qiu. "Studies on the Basic Theories for Madm." Syst Eng Theory Pract 18 (1998): 38-43.

[29] H. H. Bock,E. Diday. Analysis of Symbolic Data. Springer Berlin Heidelberg, 2000.

[30] "Implementation Rules for Comprehensive Management and Quantitative Assessment of Urban Environment During the "Twelfth Five-Year Plan"." (2011). Ministry of Ecology and Environment of the People's Republic of China. http://www.mee.gov.cn/gkml/hbb/bgth/201111/t20111116_220023.htm?keywords.

[31] "Environmental Air Quality Status Bulletin of Fujian Province." 2020 (2019). Fujian Provincial Department of Ecology and Environment. http://sthjt.fujian.gov.cn/zwgk/sjfb/hjsj/zlph/. 